\title{
SELF-HEALING SLIP PULSE ON A FRICTIONAL SURFACE
}

\author{
GILLES PERRIN $\dagger$
}

Bureau de Contrôle de la Construction Nucléaire, 15-17 Avenue Jean Bertin, 21000 Dijon, France

\author{
JAMES R. RICE and GUTUAN ZHENG \\ Department of Earth and Planetary Sciences and Division of Applied Sciences. Harvard University, \\ Cambridge, MA 02138, U.S.A.
}

(Received 19 November 1994 ; in revised form 18 March 1995)

\begin{abstract}
Guided by seismic observations of short-duration radiated pulses in earthquake ruptures, Heaton (1990) has postulated a mechanism for the frictional sliding of two identical elastic solids that consists in the subsonic propagation of a self-healing slip velocity pulse of finite duration along the interface. The same type of pulse may be conjectured for inhomogeneous slip along sufficiently large, and compliant, technological surfaces. We analyze such pulses, first as steady traveling waves which move at constant speed, and without alteration of shape, on the interface between joined elastic half-spaces, and later as transient disturbances along such an interface, arising as slip rupture propagates spontaneously from an over-stressed nucleation site. The study is conducted in the framework of antiplane elastodynamics; normal stress is uniform and alteration of it is not considered. We show that not all constitutive models allow for steady traveling wave pulses: the static friction threshold subsequent to the relocking of the fault must increase with time. That is, such solutions do not exist for pure velocity-dependent constitutive models, in which the stress-resisting slip on the ruptured surface is a continuously decreasing function of the instantaneous sliding rate (but not of its previous history or of other measures of the evolving state of the surface). Further, even for constitutive models that include both the rate- and state-dependence of friction. such as the laboratory-based constitutive models for friction as developed by Dieterich $(1979,1981)$ and Ruina (1983), steady pulse solutions do not exist for versions, like one discussed by Ruina (1983), which do not allow (rapid) restrengthening in truly stationary contact. For a particular class of rate- and statedependent laws which includes such restrengthening, we establish parameter ranges for which steady pulse solutions exist, and use a numerical method stabilized by a Tikhonov-style regularization to construct the solutions. The numerical method used for the transient analysis adopts Fourier series representations for the spatial dependence of stress and slip along the interface, with the (time-dependent) coefficients in those Fourier series being related to one another in a way which obtains from exact solution to the equations of elastodynamics. This allows an efficient numerical method, based on use of the Fast Fourier Transform in each time step, with the frictional constitutive law enforced at the FFT sample points along the interface. Solutions based on a law that includes restrengthening in stationary contact show that spontaneous rupture propagation will occur either in the self-healing slip pulse mode (but not generally as a steady pulse) or in the classical enlarging-crack mode, depending on the values of parameters which enter the constitutive law. This analysis suggests that the strictly steady, traveling wave pulse solutions may either be unstable or have a limited basin of attraction.
\end{abstract}

\section{INTRODUCTION : SELF-HEALING SLIP PULSE ON AN INFINITE FAULT SUBJECTED TO ANTIPLANE LOADING}

We consider the problem of an unbounded elastic body containing a weak plane $(y=0)$ on which frictional sliding can occur along the direction $z$. Following Heaton

† Formerly, Postdoctoral Fellow at Division of Applied Sciences, Harvard University. 
(1990) we define a steady antiplane (Mode III) slip pulse as a sliding wave, independent of coordinate $z$, propagating subsonically along the $x$ axis at constant velocity $\beta c$, where $c$ is the elastic shear wave speed of the medium, without alteration of its shape, with complete cessation of slip behind the pulse. The stress $\sigma_{y y}$ is uniform and compressive, and is unaltered by slip. The only other non-zero component of the stress tensor at infinity is $\sigma_{z y}=\sigma_{y z}=\tau_{\infty}$ (constant). We construct numerically such steady, traveling wave, elastodynamic solutions here for frictional constitutive laws of a class which allows their existence. We also introduce a new numerical procedure for transient antiplane elastodynamics, based on a spectral (Fourier series) representation of the $x$ dependence of slip. This is used to find spontaneous rupture solutions which, for appropriate constitutive laws, may involve a self-healing slip pulse mode of rupture propagation, or a mode more like that of a classical enlarging shear crack, depending on the values of constitutive parameters.

Heaton (1990) introduced such pulses to explain the short inferred duration of seismic slip at particular locations on a fault with respect to the total duration of the seismic event. He proposed such self-healing pulses as a dislocation-like alternative to conventional enlarging-crack models of rupture. In such crack models, the duration of slip at a point on the fault is comparable to the total time of propagation of the rupture event, rather than much shorter. We do not argue here the sufficiency of the observational basis for Heaton's conclusions, noting that the strongest seismic radiation is generated near the growing front of the rupture, so that it might not always be possible to distinguish the enlarging crack mode of propagation from the self-healing pulse mode. Also, for background, it may be recalled that healing pulses of short slip duration are predicted by 3D numerical elastodynamic rupture models based on simple slip-weakening friction laws (in these, strength degrades to a fixed residual level after some small slip) in circumstances for which rupture is confined to a long but narrow fault zone (Day, 1982). This mechanism of pulse generation is also seen in 2D models that approximately represent a long fault in a crustal plate that is elastically coupled to a substrate (Johnson, 1992; Myers et al., 1994). Freund's (1979) healing pulse solution in an unbounded solid, discussed by Heaton (1990), requires the ruptured surface to support more stress after locking them when sliding, while his analysis of a healing pulse in the case of geometric confinement to a narrow strip of material with clamped boundaries allows reduction of the stress supported after locking and suggests the possibility that self-healing could occur, although that feature was not demonstrated. For more equiaxed or unconfined fault zones, however, slip weakening laws lead to predictions of rupture propagation in the classical enlarging crack mode (Andrews, 1976, 1985; Day, 1982). The inferred short duration of seismic slip might also be a consequence of strong fault zone segmentation, so that rupture involves sequences of crack-like propagation over a small patch, arrest at its borders, renucleation on a neighboring patch, etc. (Papageorgiou and Aki, 1983; Boatwright, 1988). Finally, Johnson (1990) notes another mechanism of generation of healing pulses. He shows that in some circumstances a rupture may nucleate and propagate bilaterally, but arrest suddenly at a strong barrier at one end so that a healing wave, arresting slip, spreads back over the rupture surface from that end. Then the combination of the healing wave and the still-propagating other end of the rupture ultimately form a moving pulse-like configuration. 
Here, despite these other possible mechanisms, we follow the inference from Heaton's (1990) discussion that short slip duration might result from features of frictional constitutive response along essentially smooth and unbounded, homogeneous sliding surfaces. We thus ask what type of friction laws, in a class with some laboratory support, are consistent with the existence of pulses. Also, Heaton considered mode II pulses (in-plane slip, in the $x$ direction) in his modeling conjectures, whereas we address here the case of Mode III pulses. This does not bring about qualitative differences for the problem of steady propagation, at least so long as conditions are met for sub-Rayleigh wave propagation speeds in Mode II.

We begin with analysis pulses as steady traveling waves, so that the stress $\tau(x, t)$ $\left[=\sigma_{y z}(x, y=0, t)\right]$ and the sliding velocity (i.e. the displacement discontinuity rate) $V(x, t)$ take the forms $\tau=\tau(t-x / \beta c)$ and $V=V(t-x / \beta c)$. Since each point of the rupture surface experiences the same history, just shifted in time, we may focus on any particular point, say, $x=0$. It is recalled in Appendix $A$ that the following relation holds, on the basis of linear elastodynamics:

$$
\tau(t)=\tau_{x}+g \mathrm{PV} \int_{-\infty}^{+\infty} V(t+u) \frac{\mathrm{d} u}{u} .
$$

where

$$
g=\mu \sqrt{1-\beta^{2}} /(2 \pi c \beta)
$$

$\mu$ denoting the shear modulus, and the notation PV indicating that the subsequent integral is a Cauchy principal value integral. The limit $\beta=1$ corresponds to propagation at the shear wave speed. The Mode II result is of identical form with $g$ in that case being a monotonically decreasing function of propagation speed that vanishes at the Rayleigh speed, although more rapidly propagating solutions at speeds between the shear and pressure wave velocities are sometimes allowed (Andrews, 1976, 1985, 1994; Burridge et al., 1979). All of our steady Mode III solutions with $\beta<1$ have exact correspondence to sub-Rayleigh Mode II solutions.

Let us now specify the kind of frictional constitutive law which is used. We will show below that such pulse solutions do not exist for pure velocity-weakening friction laws for which the strength (called $\tau_{\max }$ below) on the ruptured part of the surface is a continuous non-increasing function of $V(t)$, and of $V(t)$ only, for $V(t) \geqslant 0$. We are thus interested in using laws of a structure that are consistent with more subtle features of laboratory experiments, in particular, with dependence of strength not only on $V(t)$ but also on the evolving state of the surface. For specific numerical illustrations, we shall use a class of rate- and state-dependent friction laws like those due to Dieterich (1979, 1981) and Ruina (1983). However, the beginning of the paper aims to establish results for a wider class of friction models. For this reason, we state here very few restrictions on the friction law, and one can easily check that the models just quoted do fulfill them. First, let us mention that those models incorporate some length scale, a characteristic slip distance for slip-weakening or evolution of state, which obliterates the crack-like singularity of sliding velocity at the rupture tip, and enforces continuity of the sliding velocity. For this reason, we assume that $V(t)$ is continuous. We also assume that the sliding velocity is always non-negative (no back-slip). The 
current stress is assumed to be limited by a maximal value which can be written as a functional of past sliding velocity

$$
\tau(t) \leqslant \tau_{\max }(t) \text { when } V(t)=0 ; \quad \tau(t)=\tau_{\max }(t) \text { when } V(t)>0 .
$$

Here,

$$
\tau_{\max }(t)=F\left[V(t) ; V\left(t^{\prime}\right),-\infty<t^{\prime}<t\right]
$$

(the notation means that $F$ is a function of the instantaneous slip rate, and a functional of its prior history) with a positive instantaneous derivative

$$
\partial F / \partial[V(t)]>0
$$

(instantaneous velocity strengthening). The functional dependence on past velocity accounts for a fading memory of previous slips. The frictional constitutive models which we consider all introduce the "state" of the surface, represented through a set of variables $\{\theta\}$, with just a single member in the simplest cases, and have the form

$$
\tau_{\max }(t)=F(V(t),\{\theta(t)\})
$$

with the evolution of the frictional state being given by a set of ordinary differential equations

$$
\mathrm{d}\{\theta(t)\} / \mathrm{d} t=\{\varphi(V(t),\{\theta(t)\})\}
$$

which ensure continuous evolution of the state variable, and hence that the instantaneous response to a velocity discontinuity be given by (5).

As a preliminary result, this instantaneous response property is used in Appendix $B$ to show that a pulse with compact support $[0, T]$ must have a specific shape at onset and arrest of slip for the relatively broad class of frictional behaviors considered up to now [(3)-(7)]. We establish there (with supporting calculations in Appendix C) the two following asymptotic behaviors:

$$
\left\{\begin{array}{l}
V(t)=\alpha t^{\gamma}+o\left(t^{\gamma}\right), t \rightarrow 0^{+}, \\
V(t)=\alpha^{\prime}(T-t)^{\gamma+1}+o\left((T-t)^{\gamma+1}\right), t \rightarrow T^{-},
\end{array}\right.
$$

where we use the $o(f(t))$ notation, representing any function $g(t)$ such that $g(t) / f(t)$ converges to 0 , where the superscript $+/-$ means a right/left limit, and where the exponent $\gamma$ amounts to

$$
\gamma=\frac{1}{2}+\frac{1}{\pi} \arctan \left(\frac{1}{\pi g} \frac{\partial F}{\partial[V(t)]}[V(t)=0]\right)
$$

and is greater than $1 / 2$ and smaller than 1 , by positiveness and finiteness of the derivative. Notice that, although our later computational results seem to deny it, the coefficients $\alpha$ and $\alpha^{\prime}$ might happen to be zero.

In Section 2, we specify two models in the rate- and state-dependent class, which we refer to as the Ruina-Dieterich model, or slip model, and the Dieterich-Ruina model or slowness model. Both share the state variable framework, but the former (Ruina, 1980, 1983 ; Dieterich, 1979) does not provide strengthening in truly stationary $(V=0)$ contact, whereas the latter (Dieterich, 1981 ; Ruina, 1980, 1983) does; 
that is, the latter is a true aging model. It might be thought that any useful friction law would have to exhibit restrengthening in truly stationary contact, but as discussed more fully in Section 2.3, Ruina (1983) showed that rate- and state-dependent laws which did not have that property could, nevertheless, closely simulate the restrengthening observed in experiments by Dieterich (1978). The matter of whether experiments do or do not show frictional restrengthening in truly stationary contact remained unclear for some time following that observation, but recent experiments (Beeler $e t$ al., 1994) covering a wide range of stiffness of the loading apparatus, and hence of the extent of relaxational slip, show that the Ruina assumption of state evolution only during slip is insufficient, and hence argue for restrengthening in stationary contact. We make it clear that this factor of restrengthening in truly stationary contact is the main feature that allows for steady propagating solutions with compact support, that is, for slip pulse solutions. For that reason, we use the Dieterich-Ruina slowness model as a basis for illustrating pulse solutions; the Ruina-Dieterich slip model is not consistent with their existence, at least as steadily traveling waves. The usual formulation of those models is not well-behaved in the vicinity of zero slip velocity; Section 3 hence introduces a regularized version of them.

In Section 4, we aim to construct solutions to the friction problem in the form of a steady propagating pulse of some duration, 7 . Let us sketch the ideas: we consider the velocity as the main unknown:

$$
V(t)=0, \quad t \leqslant 0 ; \quad V(t) \geqslant 0, \quad 0 \leqslant t \leqslant T ; \quad V(t)=0, \quad T \leqslant t .
$$

It is then possible to compute the stress $\tau^{\mathrm{IE}}(t)$ obtained from this velocity through consideration of elastodynamics only, without regard to the friction law, using the integral equation (1) (which superscript IE is meant to recall); on the other hand, one can compute the maximal stress allowed by the frictional behavior $(4), \tau_{\max }(t)$. The friction rule (3) then imposes the conditions:

$$
\tau^{\mathrm{IE}} \leqslant \tau_{\max }, \quad t \leqslant 0 ; \quad \tau^{\mathrm{IE}}=\tau_{\max }, \quad 0 \leqslant t \leqslant T ; \quad \tau^{\mathrm{IE}} \leqslant \tau_{\max }, \quad T \leqslant t .
$$

If we think of using some computational representation of the function $V(t)$, the way we have displayed the problem seems to yield the right number of equations, plus some inequalities. Yet, things are more involved, and we are in particular unable to guarantee existence and uniqueness of solutions. Moreover, when a solution exists, a simple equation solving technique happens to yield oscillatory solutions, which seems to indicate that the problem is, in some sense, ill-posed. For that reason, we resort to a minimization procedure that allows us to keep control of the first derivative of the solution. After dimensionless reduction of the problem, Section 4 expounds this algorithm and offers a discussion of the dependence of the solutions on the dimensionless parameters; it is shown that pulse propagation is possible above some loading threshold, and that the duration and shape of the pulse are fully determined. Numerical results for such steady, traveling wave, pulse solutions are provided in Section 5.

Finally, Section 6 considers the general transient elastodynamic slip problem, approached numerically based on the spectral representation of slip $\delta(x, t)$ [related to $V(x, t)$ by $V=\partial \delta / \partial t]$, mentioned earlier, as a Fourier series in $x$ that is truncated at large order. Representative numerical solutions are shown for spontaneous rupture 
propagation, with rupture nucleated by over-stressing some section of the frictional surface. We do these solutions for the regularized Dieterich-Ruina constitutive law of Section 3, and show that ruptures propagate in a classical enlarging-crack mode when a characteristic slip rate $V_{0}$ entering that law is sufficientlly low, but as a selfhealing slip pulse when $V_{0}$ is larger. Now the pulses, when found at all, are not generally steady, but may grow or decay with distance of propagation. These cracklike and pulse solutions are found at the same loading levels for which we have shown in Section 5 that a steady traveling wave pulse solution also exists, and lead us to conclude that the traveling wave solutions are either unstable or have a limited basin of attraction.

\section{OCCURRENCE OF PULSES WITHIN THE DIETERICH AND RUINA FRAMEWORK}

From now on, we specify the constitutive frictional law that we use. In particular, the "state" $\{\theta\}$ is reduced to one scalar: $\theta$.

\subsection{Ruina-Dieterich slip model; no restrengthening in truly stationary contact}

Let us first describe the Ruina-Dieterich model, which was introduced by Ruina (1983) as a simplification of an earlier model proposed by Dieterich (1979) used to fit laboratory results. Following Beeler et al. (1994), we call this the slip model, since state evolves only when there is continuing slip, $V \neq 0$. We write $\psi(t)$ for the state variable and specify the choice of Ruina $(1980,1983)$ for what we call $\tau_{\max }$ :

$$
\left\{\begin{array}{l}
\tau_{\max }(t)=\tau_{0}+A \ln \left[V(t) / V_{c}\right]+\psi(t) \\
\mathrm{d} \psi(t) / \mathrm{d} t=-[V(t) / L]\left\{\psi(t)+B \ln \left[V(t) / V_{c}\right]\right\} .
\end{array}\right.
$$

The quantities $\tau_{0}, A, B, V_{c}$ and $L$ are constants. More precisely, $L$ is a slip length scale for state evolution; $A$ and $B$, which are positive, account respectively for the shorttime velocity strengthening and for the steady-state velocity weakening. Sometimes (e.g. Beeler et al., 1994) (12) is written with a variable $\theta$, with $\psi=\ln \left(V_{c} \theta / L\right)$. It is possible to introduce in this model an arbitrary number of state variables, $\left(\psi_{i}\right)_{i=1 . \ldots}$, each of them having specific weakening constant $B_{i}$ and length scale $L_{i}$. For example, Ruina $(1980,1983)$ and Weeks and Tullis (1985) have used two state variables to fit, in different contexts, their experimental data, and Gu et al. (1984) studied the non-linear stability problem for a one-degree-of-freedom system based on such models.

\subsection{Dieterich-Ruina slowness model; restrengthening in truly stationary contact}

Let us now describe the Dieterich-Ruina model, based on writing calculational procedures of Dieterich in an explicit state variable form (Ruina, 1980). Following Ruina (1983) and Beeler et al. (1994), this is also called the slowness law; it includes true aging. The law is used in Dieterich $(1981,1986,1992)$ and Okubo (1989); Okubo 
and Dieterich (1986) use a slightly different version of it. The Dieterich-Ruina model consists in choosing

$$
\left\{\begin{array}{l}
\tau_{\max }(t)=\tau_{0}-A \ln \left[1+V_{\infty} / V(t)\right]+B \ln \left[1+\theta(t) / \theta_{0}\right], \\
\mathrm{d} \theta(t) / \mathrm{d} t=1-\theta(t) V(t) / L
\end{array}\right.
$$

One might think of the state variable $\theta$ here in an abstract way as Ruina $(1980,1983)$ does, especially in connection with (12), but Dieterich (1979), and Dieterich and Conrad (1984), interpret it as the "average age of the load supporting contacts between the sliding surface". In that case a constitutive law of the form (13) is more sensible than one of the form (12), since it yields $\mathrm{d} \theta / \mathrm{d} t=1$ for $V=0$. That contact time interpretation led Dieterich to use extensively equations (13), although equation $\left(13_{2}\right)$ seems to have been first written by Ruina (1980). The quantities $\tau_{0}, A, B, V_{x}$, $\theta_{0}$ and $L$ are constants, with the same general meaning as formerly, except that $V$, and $\theta_{0}$ are cut-offs for high velocity and short contact duration.

\subsection{Occurrence of a pulse}

In this section, we examine whether the preceding friction laws allow for slip-pulse solutions. We also show that classical laws of a pure velocity-dependent type do not allow slip-pulse solutions.

Let us stress that the two models just described are much akin to each other. In particular, they share a low velocity divergence because of the term $\log [V(t)]$. This can be fixed by introducing some low velocity cut-off, $V_{0}$, and replacing that term by $\log \left[V_{0}+V(t)\right]$. We will not say more about it now, and fully address this regularization in Section 3 for the Dieterich-Ruina model. Before that time, let us just ignore the errant behavior of the function $F(V, \theta)$ for $V=0$, assuming it has been regularized there.

Here, we are concerned with the only qualitative difference between the RuinaDieterich model (Section 2.1) and the Dieterich-Ruina model (Section 2.2), which lies in that, for a non-slipping fault, the threshold $\tau_{\max }(t)$ remains stuck at its value at arrest, $\tau_{\max }(T)$, in the former, whereas it climbs upwards, $\tau_{\max }(t)>\tau_{\max }(T)$ in the lattcr. Ruina $(1980,1983)$ considers this as a drawback of his model, but sensibly alleges that the low velocity behavior is however non-measurable from the experiments he and Dieterich performed. More precisely, these experiments consisted of measuring the frictional force on a sliding surface with a driven velocity, imposed elsewhere in an elastically deformable system (e.g. as oil inflow to a hydraulic cylinder), which is a piece-wise constant function of time. In particular, Dieterich (1978) measured the force in an experiment where, after previous sliding, the driving was stopped from time $-t$ to 0 , and resumed with a uniform non-zero velocity for positive time. The force increases to some maximal value and afterwards decreases. Dieterich found that its peak value, interpreted as the static friction, for a particular quartzite varied with waiting time, $t$, approximately as

$$
\tau_{\text {static }}=\left\{0.55+0.02 \log _{10}[1+t / \mathrm{s}]\right\} \sigma
$$

where $\sigma$ is the normal stress. But Ruina (1983) noted the following. First, what is controlled in Dieterich's experiments is not the motion of the slip surface, but the 
motion of some load point, connected to the sliding surface by means of some deformable device, which can be modeled as a spring. Second, the load point was moved at constant rate, $1 \mu \mathrm{m} / \mathrm{s}$, then stopped for successively longer times, $t$, and motion was afterwards resumed at the original load point speed. Ruina remarks that these procedures do not imply that the surface is motionless during the "waiting" period. Specifically, Ruina (1983) considers a spring rigidity consistent with Dieterich's (1978) experiments, assumes that the friction law is of a form similar to (12) (but with two state variables, with evolution laws that he estimated independently from "velocity jump" tests), and numerically integrates the set of equations. Although the friction law would predict no change of state for $V=0$, the computed static shear stress fits very well Dieterich's (1978) empirical relation (14) [see Fig. 6 of Ruina (1983)]. Ruina (1983) concludes that, in the computation, "the apparent static friction is due entirely to small amounts of slip that take place while the load point is still, not [due to] time of contact." He adds, however : "I do not want to claim that no healing is ever possible when there is no slip, but that static friction cannot necessarily be characterized by a single number like time of stationary contact". This discussion has remained unsettled up to the time of experiments described by Beeler et al. (1994), which studied the restrengthening during relaxational slip over a wide range of elastic stiffness of the loading apparatus. Smaller stiffness means greater relaxational slip. The Beeler et al. (1994) results are fit only moderately better by the Dieterich-Ruina slowness law than by the Ruina-Dieterich slip law, but they do suggest that the assumption that state evolves only during continuing slip is an inadequate one, and argue for evolution of our $\tau_{\max }$ in truly stationary contact.

When one wants to apply a model of the Dieterich and Ruina framework to examine the possibility of self-healing pulses, the behavior of $\tau_{\max }(t)$ after slip arrest is of paramount importance. More precisely, a pulse cannot occur in the steadily traveling wave context considered if $\tau_{\max }(t)$ remains stuck when velocity returns to zero, which we show by reductio ad absurdo. Suppose that, in the Ruina-Dieterich model, there exists a pulse-like solution, that is a continuous slip velocity with support $[0, T]$. On the one hand, from the constitutive law, because the state variable remains frozen from $t=T$ on $\left(12_{2}\right)$, because both $\tau_{\max }$ and $\tau^{\mathrm{IE}}$ are continuous, and because the velocity is positive at time arbitrarily near $T$, we have

$$
\tau_{\max }(t)=\tau_{\max }(T)=\tau^{\mathrm{IE}}(T) \quad \text { for } t \geqslant T .
$$

On the other hand, from the elastodynamic integral equation, for $t>T$,

$$
\tau^{\mathrm{IE}}(t)-\tau_{\max }(t)=\tau^{\mathrm{IE}}(t) \quad \tau^{\mathrm{IE}}(T)=(t-T) g \int_{0}^{T} \frac{V(u)}{(t-u)(T-u)} \mathrm{d} u>0
$$

since $V \geqslant 0$. This inequality is contradictory with the requirement

$$
\tau_{\max }(t) \geqslant \tau^{\mathrm{IE}}(t),
$$

which thus proves that a slip-pulse solution does not exist in the absence of restrengthening in truly stationary contact.

While the argument has been given in terms of rate- and state-dependent friction laws, its conclusion is readily extended to laws in which friction is purely rate- 
dependent. For such laws, $\tau_{\max }$ is a unique and, we assume, continuous function of $V$ of velocity weakening type (i.e. $\mathrm{d} \tau_{\max } / \mathrm{d} V \leqslant 0$ ), for $V \geqslant 0$ and hence $\tau_{\max }$ does not change with time when $V=0$. That is, the laws do not allow further strengthening in stationary contact. The same argument as above leads to the inconsistent inequalities in $\tau_{\max }$ and $\tau^{\mathrm{IE}}$, and hence disallows a slip-pulse solution. Two further comments are in order : first, a pure velocity-dependent law contains no length scale and thus allows for no feature like a slip-weakening zone or fracture energy at the advancing front of the rupture. Any propagating slip distribution would then have to move at the shear wave speed (or Rayleigh speed in Mode II) to avoid a singularity of stress at the advancing edge. Thus, when we refer here to pure velocity-dependent laws, we include the case for which some special feature, e.g. a fracture energy allowing for singular stress ahead of the rupture, is invoked to describe the start of rupture, and for which the pure velocity-dependent law applies once the rupture front has passed and slip has begun. Our proof that no slip-pulse solution exists assumes only that $V \geqslant 0$ everywhere and that the friction law makes $\tau_{\max }$ a continuous function of $V$ only, at least in the vicinity of the hypothesized trailing edge where slip is arrested. Second, our non-existence result can be avoided if $\tau_{\max }(V)$ is discontinuous at $V=0$ and if $\tau_{\max }(0)\left(=C\right.$, say) is then sufficiently greater than $\tau_{\max }(0+)(=D$, say). However, such is a mathematically questionable form of a pure velocity-dependent law, in that conclusions drawn from it (the existence of a pulse solution) are lost as soon as the discontinuous $\tau_{\max }(V)$ is "regularized" to one which changes rapidly but continuously with $V$ near $V=0$, from $\tau_{\max }=C$ at $V=0$ to $\tau_{\max } \approx D$ at small $V>0$. The regularized form disallows a slip-pulse solution.

Thus to the extent that Heaton's (1990) seismic observations credibly support his inference of traveling slip-pulse-like solutions, they might be regarded as arguing for restrengthening in truly stationary contact. The existence of Heaton pulses would also provide further evidence for the physical inadequacy of using pure velocitydependent friction laws, without additional dependence on the evolving state of the surface, for modeling frictional slip between elastic continua; we have noted above that such laws are inconsistent with the existence of pulses. The computational part of this study, in the subsequent sections, deals only with the Dieterich-Ruina slowness model which, of the models considered here, allows slip-pulse solutions to exist in some parameter ranges. Tullis et al. (1992) and Beeler and Tullis (1995) have done numerical studies of spontaneous dynamic rupture in which slip evolves with position and time, analogous to what we present in Section 6 here, for the in-plane (Mode II) case. They use a boundary integral program devised by Andrews (1985) and previously adapted to the rate- and state-dependent context by Okubo (1989), and find consistent results in that the simulations which evolve into a slip-pulse mode of rupture propagation are those which are based on laws allowing strengthening in truly stationary contact.

\section{A REGULARIZED VERSION OF DIETERICH-RUINA MODEL}

As pointed out by Dieterich (1992), the model presented offers non-physical behavior for extremely low slip velocities. It does the same for (perhaps unreasonably) 
long "contact times" $\theta$. To overcome these drawbacks, we introduce two cut-off velocities $V_{0}$ and $V_{1}$ in the following way:

$$
\left\{\begin{array}{l}
\tau_{\max }(t)=\tau_{0}+A \ln \left\{\left[V_{0}+V(t)\right] /\left[V_{\infty}+V(t)\right]\right\}+B \ln \left[1+\theta(t) / \theta_{0}\right], \\
\mathrm{d} \theta(t) / \mathrm{d} t=1-\theta(t)\left[V_{1}+V(t)\right] / L
\end{array}\right.
$$

Notice that the state variable is confined in $\left[0, L / V_{1}\right]$. This might not seem wise if $\theta$ is to be interpreted as a contact time. However, considering a cut-off precisely means that we are getting outside of the measurable range and is by nature artificial. Another way of regularizing the Dieterich-Ruina model will be briefly discussed in Section 5 . The cut-off velocities just introduced seem to be arbitrary. Still, let us consider the case of a steady state friction with velocity $V$; then

$$
\tau_{\max }=\tau_{0}+A \ln \left\{\left[V+V_{0}\right] /\left[V+V_{\infty}\right]\right\}-B \ln \left\{\left[V+V_{1}\right] /\left[V+V_{1}+L / \theta_{0}\right]\right\},
$$

which suggests that there would be two different physical cut-off mechanisms, at low as well as at high velocities, unless we choose

$$
V_{1}=V_{0} \text { and } \quad V_{1}+L / \theta_{0}=V_{\infty},
$$

which we do. The regularized Dieterich-Ruina model which we shall consider in the following is thus given by equations

$$
\left\{\begin{array}{l}
\tau_{\max }(t)=\tau_{0}+A \ln \left\{\left[V_{0}+V(t)\right] /\left[V_{\infty}+V(t)\right]\right\}+B \ln \left[1+\theta(t)\left(V_{\infty}-V_{0}\right) / L\right], \\
\mathrm{d} \theta(t) / \mathrm{d} t=1-\theta(t)\left[V_{0}+V(t)\right] / L
\end{array}\right.
$$

\section{PULSES WITH THE REGULARIZED DIETERICH-RUINA MODEL : ALGORITHMS}

\subsection{Choice of dimensionless parameters}

If it can be solved, the mathematical problem will yield a solution $(V(t), \tau(t), \theta(t))$ which depends on 12 parameters and on the number $N$ of discretization elements used which sets its accuracy. The 12 parameters can be sorted into three families: constitutive parameters of the elastic medium and of the friction rule $\left(\mu, c, \tau_{0}, A, B\right.$, $L, V_{0}, V_{\infty}$ ), initial state and loading of the fault (initial value of the state variable $\left.\theta(0), \tau_{\infty}\right)$ and response of the fault (duration $T$, velocity $\beta c$ of the pulse).

A first remark is that $\theta(0)$ is in fact not free if a strict traveling wave solution, with $x-\beta c t$ dependence, is to result: indeed, if it is different from $L / V_{0}, \theta$ tends exponentially towards $\pm \infty$ for $t \rightarrow-\infty$, which is unpleasant. For this reason, we fix $\theta(0)=L / V_{0}$, which is reasonably assumed for a surface which has been in contact without sliding for a much longer time than that of typical laboratory studies.

Now, analysis of the way the mathematical problem varies with the remaining parameters allows an attempt to solve it for only five free reduced parameters: $\hat{V}_{\infty}$ and $\hat{A}$ related to the friction rule, $\hat{\tau}_{\infty}$, related to the loading, and $\hat{T}$ and $\hat{\beta}$, related to the pulse duration and velocity. More precisely, we consider the problem, derived from (1), (2) and (15), 


$$
\begin{gathered}
\hat{\tau}(\hat{t})=\frac{1}{\hat{\beta}} \mathrm{PV} \int_{0}^{1} \frac{\hat{V}(u)}{u-\hat{t}} \mathrm{~d} u, \\
\left\{\begin{array}{l}
\hat{\tau}_{\max }(\hat{t})=-\hat{\tau}_{\infty}+\hat{A} \ln \left\{[1+\hat{V}(\hat{t})] /\left[\hat{V}_{\alpha}+\hat{V}(\hat{t})\right]\right\}+\ln \left[1+\hat{T} \hat{\theta}(\hat{t})\left(\hat{V}_{x}-1\right)\right], \\
\mathrm{d} \hat{\theta}(\hat{t}) / \mathrm{d} \hat{t}=1-\hat{T} \hat{\theta}(\hat{t})[1+\hat{V}(\hat{t})]
\end{array}\right.
\end{gathered}
$$

together with the transcription [denoted $(\hat{3})$ hereafter] of system (3) to variables with $a^{\wedge}$, the solution of which is related to the physical solution through :

$$
\begin{gathered}
V_{x}=V_{0} \hat{V}_{x}, \quad A=B \hat{A}, \quad \tau_{\infty}=\tau_{0}+B \hat{\tau}_{x}, \quad T=L \hat{T} / V_{0}, \\
\beta / \sqrt{1-\beta^{2}}=\mu V_{0} \hat{\beta} /(2 \pi c B), \quad t=L \hat{T} \hat{t} / V_{0}, \quad V(t)=V_{0} \hat{V}(\hat{t}), \\
\left.\tau^{\cdot}(t)=\tau_{0}+B\left(\tau^{\cdot}(\hat{t})+\hat{\tau}_{\infty}\right) \text { (both for } \tau^{\cdot} \equiv \tau^{\mathrm{IE}} \text { and } \tau^{\cdot} \equiv \tau_{\text {max }}\right), \quad \theta(t)=L \hat{T} \hat{\theta}(\hat{t}) / V_{0} .
\end{gathered}
$$

In these fomulae, $\mu, c, \tau_{0}, B, V_{0}$ and $L$ are fixed materials parameters ; they will depend on the temperature (not considered here) and at least $\tau_{0}$ and $B$ will depend (linearly if Amonton-Coulomb-like) on the normal stress on the fault, constant here.

\subsection{A tentative numerical algorithm}

The aim of this sub-section is to construct a simple algorithm which hopefully will find a solution, at least if any exists, of the dimensionless problem for given $\hat{V}_{x}, \hat{A}$, $\hat{\tau}_{\infty}, \hat{T}$ and $\hat{\beta}$.

We follow the scheme sketched in the introduction. The first step is to discretize the velocity as a piece-wise constant function in the following way: we cut the interval $[0,1]$ into $N$ consecutive sub-intervals of length $\Delta \hat{t}_{i}$, the centers of which are denoted $\hat{t}_{i}$, on which the velocity is

$$
\hat{V}_{i}=\frac{i(N+1-i)^{\gamma+1}}{\sum_{j=1}^{N} j^{\prime}(N+1-j)^{\gamma+1}} d_{i}, \quad \text { where } \gamma=\frac{1}{2}+\frac{1}{\pi} \arctan \frac{\hat{A}\left(1-1 / \hat{V}_{x}\right) \hat{\beta}}{\pi} \text {. }
$$

the $\left(d_{i}\right)_{1 \leqslant i \leqslant N}$ being considered as the fundamental degrees of freedom; the sub-interval lengths are chosen uniform both in the vicinity of $\hat{t}=0$ and of $\hat{t}=1$, which ensures at both pulse tips, without singular variation of the $\left(d_{i}\right)_{1 \leqslant i \leqslant N}$, the power law behaviors noted in Section 1 and Appendices B and C. The second step is to compute the value of

$$
\hat{\tau}^{\mathrm{IE}}(\hat{t}) \equiv \frac{1}{\hat{\beta}} \sum_{j=1}^{N} \hat{V}_{j} \ln \left|\frac{\hat{t}_{j}+\Delta \hat{t_{j}} / 2-\hat{t}}{\hat{t}_{j}-\Delta \hat{t}_{j} / 2-\hat{t}}\right|
$$

at the center $\hat{t}_{i}$ of the intervals. Such choice of collocation points for (17) allows us to address the problem despite the discontinuous numerical representation of the velocity. The third step is to integrate analytically $(18)$ to get $\tilde{\tau}_{\max }\left(\hat{t}_{i}\right)$ (this is straightforward, since $\hat{V}$ is assumed to be piece-wise constant). The fourth step is to find values of $\left(d_{i}\right)_{1 \leqslant i \leqslant N}$ which ensures that $(\hat{3})$ is fulfilled, both inside interval $[0,1]$ and outside of it. 
To perform step four, we first tried to find the solution of $(\hat{3})$ by means of a classical Newton method. Two problems appear:

(i) Convergence is not realized : the family $\left(d_{i}\right)_{1 \leqslant i \leqslant N}$, initially uniform, turns wavy and even chaotic in a few cases.

(ii) Besides, it is to be expected that, once the characteristic of the medium and the loading $\hat{\tau}_{\infty}$ are known, there is only one (or a few isolated) pulse solutions possible; knowledge of the loading conditions hence determines, among other things, the pulse duration and the pulse velocity, or in other terms the couple $(\hat{T}, \hat{\beta})$. If this intuition is true, the dimensionless problem, in the way we have stated it, would almost never have a solution. In other words, for given characteristics of the medium $\hat{A}$ and $\hat{V}_{\infty}$, we expect that the set of values of $\left(\hat{\tau}_{\infty}, \hat{T}, \hat{\beta}\right)$ for which a solution exists lie along a curve in three-dimensional space.

One way to overcome problem (ii) would be to consider the couple $(\hat{T}, \hat{\beta})$ as two additional unknowns and add them in the Newton formulation. However, this improvement did not solve problem (i) : indeed, even when the couple $(\hat{T}, \hat{\beta})$ was set to a value consistent with $\hat{\tau}_{\infty}$, oscillatory divergence of the solution was observed.

\subsection{A suitable numerical algorithm}

As a resolution, we address primarily problem (i) with a regularization technique inspired by Tikhonov procedures (Tikhonov and Goncharsky, 1987). The solution to problem (ii) will follow from use of the technique. The previously decribed steps one, two and three are unchanged.

Regarding step four, we consider that the oscillations observed are a sign that some additional regularity condition should be imposed on the solution. We hence turn the solving of equations $(\hat{3})$ into a minimization problem : we define the criteria

$$
J_{1}=\sum_{i=1}^{N} \Delta \hat{t}_{i}\left(\hat{\tau}^{\mathrm{IE}}\left(\hat{t}_{i}\right)-\hat{\tau}_{\max }\left(\hat{t}_{i}\right)\right)^{2}, \quad J_{2}=\frac{1}{N-1} \sum_{i=1}^{N-1}\left(\frac{d_{i+1}-d_{i}}{1 / N}\right)^{2}, \quad J_{\rho}=J_{1}+\rho J_{2}, \quad \rho>0,
$$

[notice that $J_{1}=0$ is equivalent to fulfillment of $(\hat{3})$ ] and we denote by $J\left(\rho, N, \hat{V}_{\infty}\right.$, $\hat{A}, \hat{\tau}_{\infty}, \hat{T}, \hat{\beta}$ ) the minimal value of $J_{\rho}$ over all positive $\left(d_{i}\right)_{1 \leqslant i \leqslant N}$ (for simplicity, this set will now be denoted $\{d\})$ and $\left\{d^{*}\right\}\left(\rho, N, \hat{V}_{\infty}, \hat{A}, \hat{\tau}_{\infty}, \hat{T}, \hat{\beta}\right)$ the value of the degrees of freedom for which the minimum is reached. In that way, we keep a control of the regularity of $d_{i}$ as a function of $i$, this control being all the stronger as $\rho$ is increased. On the other hand, too large a $\rho$ diverts us from the physical problem. The idea here is that, because $N$ is finite, the problem of minimization of $J_{1}$ does not make sense. In non-computational words, it is compulsory to take the limit $N \rightarrow \infty$ before the limit $\rho \rightarrow 0$, or at least to make $N$ large enough before reducing $\rho$, and the numerical solution then regulary converges towards the smooth physical solution

$$
\{\vec{d}\}\left(\hat{V}_{\infty}, \hat{A}, \hat{\tau}_{\infty}, \hat{T}, \hat{\beta}\right)=\lim _{\rho \rightarrow 0^{+}}\left[\lim _{N \rightarrow \infty}\left\{d^{*}\right\}\left(\rho, N, \hat{V}_{\infty}, \hat{A}, \hat{\tau}_{\infty}, \hat{T}, \hat{\beta}\right)\right]
$$

of the limit minimization problem 


$$
\bar{J}\left(\hat{V}_{\infty}, \hat{A}, \hat{\tau}_{\infty}, \hat{T}, \hat{\beta}\right)=\lim _{\rho \rightarrow 0^{+}}\left[\lim _{N \rightarrow \infty}\left[\min _{\left\{d_{j} \geqslant 0\right.} J_{p}(\{d\})\right]\right] .
$$

This solves the smoothness issue. However, the solution of problem (19) is a solution to our physical (dimensionless) problem $(17,18, \hat{3})$ only in the case when the solution of (19) also fulfills $\bar{J}=0$. This is generally not the case for arbitrary $\hat{V}_{\infty}, \hat{A}, \hat{\tau}_{x}, \hat{T}$ and $\hat{\beta}$, and the problem (ii) raised at the end of Section 5.2 is not solved yet.

The way to solve it, however, is now straightforward: we must tune the five parameters to solve the equation

$$
\bar{J}\left(\hat{V}_{\infty}, \hat{A}, \hat{\tau}_{\infty}, \hat{T}, \hat{\beta}\right)=0 .
$$

The nicest way would be to use the Newton method in the five variables. However, it is a long (computational) way to $\bar{J}$, and even its first derivatives are too irregular to give a good result. Instead, a more basic but robust method, of mere scanning, was used. In the end, for various given values of $\hat{V}_{\infty}, \hat{A}$ and $\hat{\beta}$, we observed that we were able to (and that we had to) tune $\hat{\tau}_{\infty}$ and $\hat{T}$ to fulfill (20), therefore following the mentioned curve in the three-dimensional space of $\left(\hat{\tau}_{x}, \hat{T}, \hat{\beta}\right)$.

\section{PULSES WITH THE REGULARIZED DIETERICH-RUINA MODEL: COMPUTATIONAL RESULTS}

The problem was first studied intensively for a set of values for the materials parameters :

$$
\hat{A}=0.1, \quad \hat{V}_{\infty}=10^{3} .
$$

Then some computations were also performed for

$$
\hat{A}=0.2, \quad \hat{V}_{\infty}=10^{3} \text {. }
$$

We have made computations with $N=50$ and $N=100$. The latter proved to be a good trade-off between computation time and accuracy. Now, for a given value of the penalty parameter $\rho$ (say $10^{-8}$ ), when scanning the threc-dimensional space $\left(\hat{\tau}_{x}\right.$, $\hat{T}, \hat{\beta}$ ) and computing $\bar{J}$, one realizes that this non-negative function is rather small in a region that follows a curve, like a rope of slowly varying section, and offers large gradients in the section plane. This feature is all the more pronounced when $N$ is larger. When $\rho$ is diminished, the minimal value is somewhat lowered, but, if $\rho$ is too small, the rope region becomes unclear, and the solution, in terms of $d_{i}$ as a function of $i$, becomes unstable; our method might even not be able to find a minimum anymore, so that apparently $\bar{J}$ sometimes increases with $\rho$ decreasing! In practice, $\rho$ was adjusted along the rope to get a good tradc-off between a minimal value for $J$ and a smooth solution. Figure 1 shows the degrees of freedom $(\{d\})$ for each of the 15 computed solutions in the case $\hat{A}=0.1$. The regularity of their behavior for low $i$ and for high $i$ (corresponding to the tips of the pulse) is a good verification of the power law behavior of $\hat{V}(\hat{t})$ predicted analytically in (8).

Figure 2 shows $\hat{V}(\hat{t}), \hat{\tau}_{\max }(\hat{t})$ and $\hat{\tau}^{\mathrm{EE}}(\hat{t})$ for three of the solutions we constructed, corresponding to the lowest (4.623), intermediate (5.519) and highest (6.175) value 


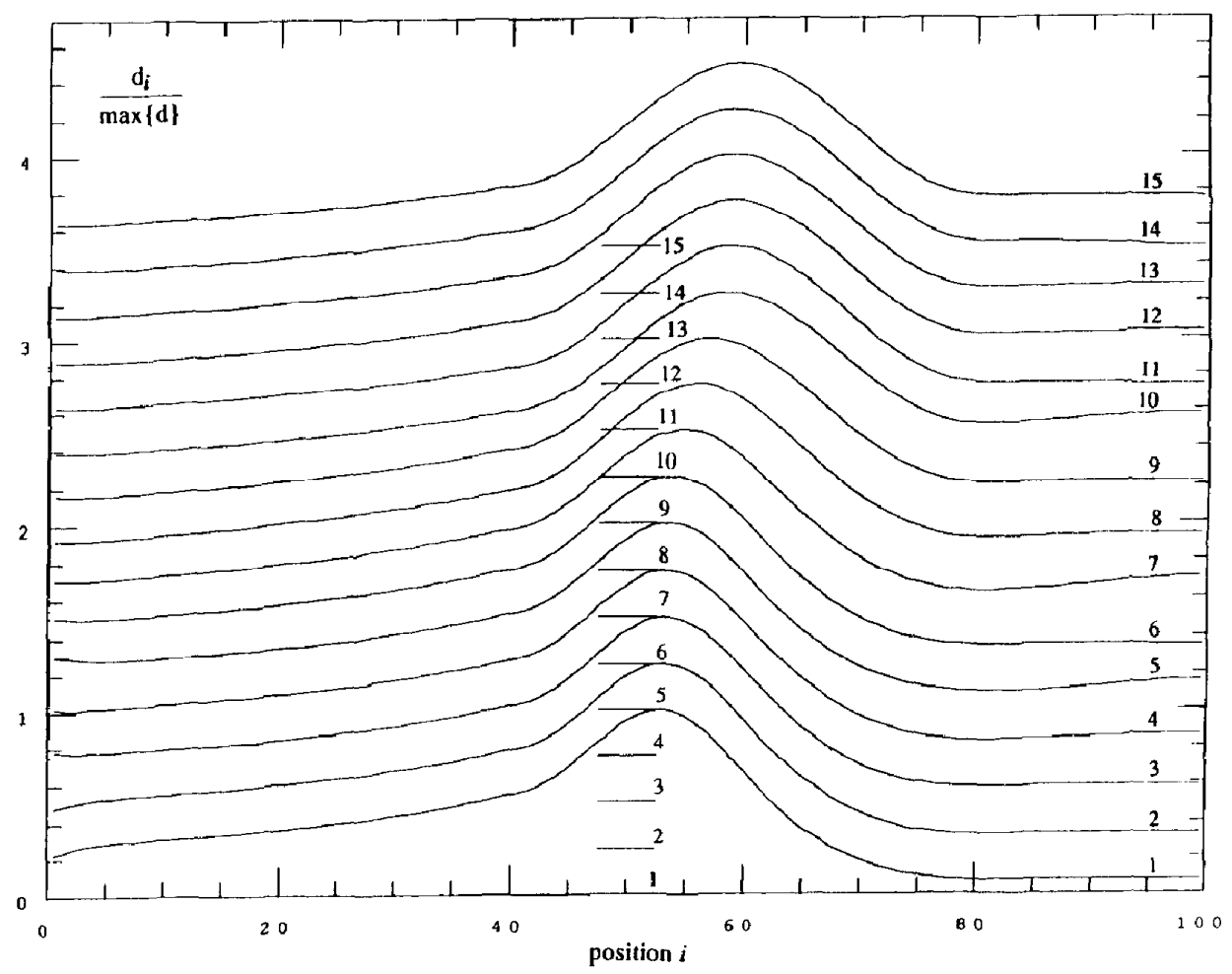

Fig. 1. Display of degrees of freedom $\left(d_{i}\right)_{i=1 \ldots 100}$ for each of the 15 solutions computed in the case $\hat{A}=0.1$. Each curve is normalized by division by its maximal $d_{i}$, and shifted vertically so that they do not cross; for each curve, the horizontal segment shows the origin of the vertical axis.

of the remove stress $\hat{\tau}_{\infty}$. Table 1 lists all the solutions which we computed for the case $\hat{A}=0.1$, sorted by increasing remote stress $\hat{\tau}_{\infty}$. For all the solutions, the slip velocity increases towards a peak value $\hat{V}_{\max }$, and more gradually decreases towards 0 . We also computed the total amount of slip caused by the pulse, that is

$$
\hat{\delta}_{\text {total }}=\int_{0}^{1} \frac{\mathrm{d} \hat{\delta}}{\mathrm{d} \hat{t}} \mathrm{~d} \hat{t}=\int_{0}^{1} \hat{V}(\hat{t}) \mathrm{d} \hat{t} .
$$

One can use it to compute the amount of work dissipated by friction per unit of crack length and of material thickness by the passing of the pulse. In terms of dimensioned quantities it amounts to

$$
D=\int_{-\infty}^{\infty} \tau(t) V(t) \mathrm{d} t
$$

which can easily be related to the dimensionless quantities as

$$
D=L \tau_{0}\left(\hat{T} \hat{\delta}_{\text {total }}\right)+L B\left(\hat{\tau}_{\infty} \hat{T} \hat{\delta}_{\text {total }}\right)
$$

because (17) makes the integral over dimensionless time of the product $\hat{\tau}(\hat{t}) \hat{V}(\hat{t})$ 
Self-healing slip pulse

(a)

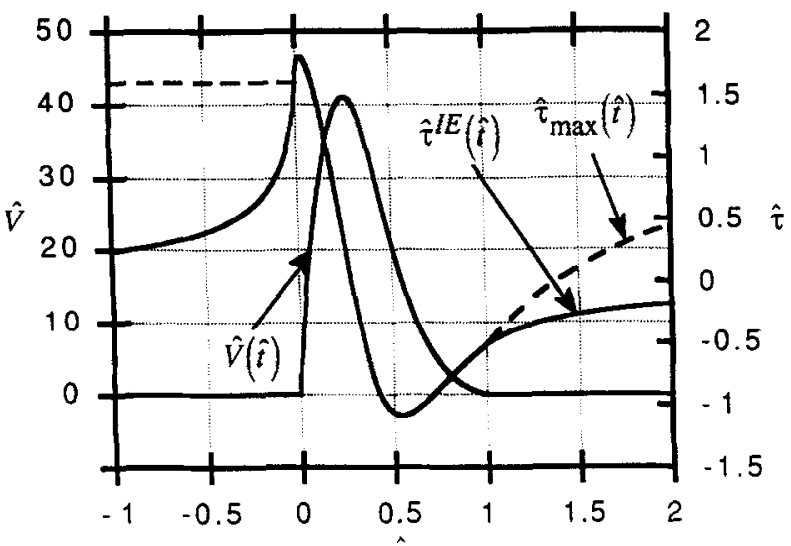

(b)

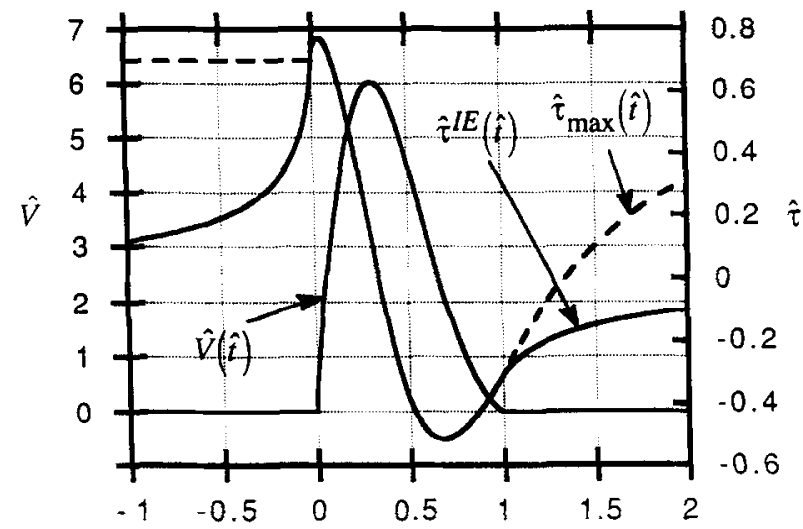

(c)

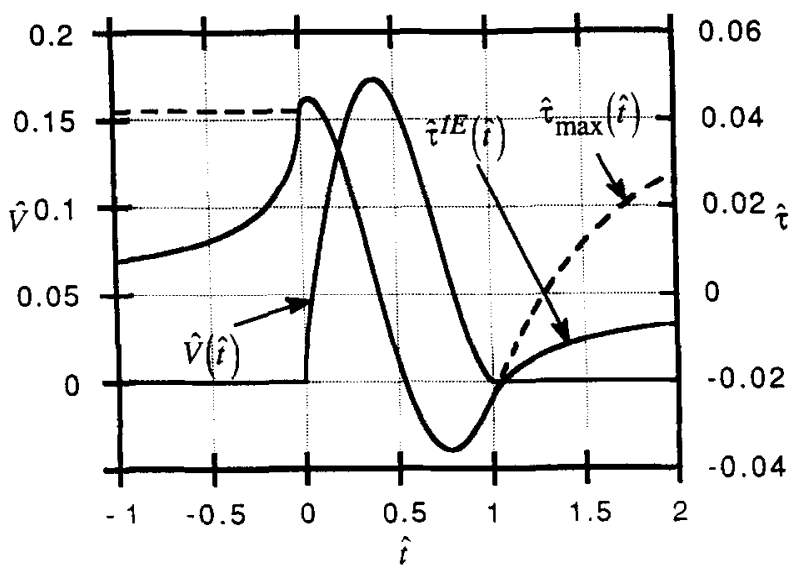

Fig. 2. Solution computed for $\hat{A}=0.1, \hat{V}_{x}=1000$ and (a) $\hat{\tau}_{x}=4.623$, (b) $\hat{\tau}_{x}=5.519$, and (c) $\hat{\tau}_{x}=6.175$.

vanish. Table 1 also provides the values of the dimensionless parentheses appearing in (21). Table 2 gives analogous information in the case $\hat{A}=0.2$.

It must be stressed that the finding of triplets $\left(\hat{\tau}_{\infty}, \hat{T}, \hat{\beta}\right)$ which allow for minimum 
Table 1. $\hat{\mathrm{A}}=0.1$

\begin{tabular}{|c|c|c|c|c|c|c|c|c|}
\hline Index & $\hat{\tau}_{\infty}$ & $\hat{T}$ & $\hat{\beta}$ & $\hat{V}_{\max }$ & $\begin{array}{c}J_{1} / \hat{V}_{\max }^{2} \\
\left(\times 10^{-8}\right)\end{array}$ & $\hat{\delta}_{\text {total }}$ & $\hat{T} \hat{\delta}_{\text {total }}$ & ${ }_{t \infty} \hat{T} \hat{\delta}_{\text {total }}$ \\
\hline 1 & 4.623 & 0.252 & 60.0 & 41.4 & 0.058 & 18.46 & 4.652 & 21.51 \\
\hline 2 & 4.692 & 0.271 & 55.0 & 36.0 & 0.23 & 16.46 & 4.461 & 20.93 \\
\hline 3 & 4.7685 & 0.295 & 50.0 & 31.1 & 0.59 & 14.42 & 4.254 & 20.28 \\
\hline 4 & 4.798 & 0.3063 & 48.0 & 29.3 & 0.097 & 13.65 & 4.181 & 20.06 \\
\hline 5 & 4.848 & 0.325 & 45.0 & 26.4 & 0.66 & 12.45 & 4.046 & 19.62 \\
\hline 6 & 4.9435 & 0.362 & 40.0 & 21.9 & 0.22 & 10.47 & 3.790 & 18.74 \\
\hline 7 & 5.178 & 0.4662 & 30.0 & 13.4 & 0.22 & 6.761 & 3.152 & 16.32 \\
\hline 8 & 5.519 & 0.6896 & 20.0 & 6.03 & 0.15 & 3.188 & 2.198 & 12.13 \\
\hline 9 & 5.769 & 0.916 & 15.0 & 2.91 & 6.1 & 1.600 & 1.466 & 8.455 \\
\hline 10 & 6.02475 & 1.226 & 11.2 & 0.938 & 6.2 & 0.5431 & 0.6658 & 4.012 \\
\hline 11 & 6.097 & 1.356 & 10.3 & 0.539 & 4.7 & 0.3115 & 0.4224 & 2.575 \\
\hline 12 & 6.1053 & 1.3645 & 10.2 & 0.497 & 4.7 & 0.2889 & 0.3942 & 2.407 \\
\hline 13 & 6.114 & 1.378 & 10.1 & 0.453 & 4.4 & 0.2644 & 0.3643 & 2.228 \\
\hline 14 & 6.151 & 1.44 & 9.7 & 0.280 & 11.0 & 0.1638 & 0.2359 & 1.451 \\
\hline 15 & 6.175 & 1.495 & 9.4 & 0.173 & 4.7 & 0.1013 & 0.1514 & 0.935 \\
\hline
\end{tabular}

Table 2. $\hat{\mathrm{A}}=0.2$

\begin{tabular}{clllccccc}
\hline Index & $\hat{\tau}_{\infty}$ & \multicolumn{1}{c}{$\hat{T}$} & $\hat{\beta}$ & $\hat{V}_{\max }$ & $\begin{array}{c}J_{1} / \hat{V}_{\max }^{2} \\
\left(\times 10^{-8}\right)\end{array}$ & $\hat{\delta}_{\text {total }}$ & $\hat{T} \hat{\delta}_{\text {total }}$ & $\hat{\tau}_{\infty} \hat{T} \hat{\delta}_{\text {total }}$ \\
\hline 1 & 4.525 & 0.55 & 30.85 & 19.92 & 0.7 & 7.115 & 3.91 & 17.71 \\
2 & 4.7 & 0.70 & 24.0 & 10.24 & 2.4 & 4.721 & 3.30 & 15.53 \\
3 & 4.8 & 0.777 & 20.76 & 7.778 & 1.8 & 3.798 & 2.95 & 14.16 \\
4 & 4.98 & 0.914 & 15.0 & 3.600 & 2.5 & 1.874 & 1.71 & 8.53 \\
5 & 5.4 & 1.81 & 9.1315 & 0.587 & 1.0 & 0.3321 & 0.601 & 3.25 \\
6 & 5.478 & 2.049 & 8.235 & 0.200 & 5.0 & 0.1160 & 0.238 & 1.302 \\
\hline
\end{tabular}

$J_{1}$ is tedious. This makes the numbers given in the corresponding columns of Tables 1 and 2 valuable. Graphic presentation of these quantities (together with $\hat{V}_{\max }$ ) is interesting too. Figs 3, 4 and 5, respectively, show evolution of $\hat{T}, \hat{\beta}$ and $\hat{V}_{\max }$ versus $\hat{\tau}_{\infty}$ for $\hat{A}=0.1$, and $\hat{A}=0.2$.

\subsection{Discussion of steady pulse solutions}

These curves suggest that slip-pulse solutions exist only for

$$
\hat{\tau}_{\infty}^{-}\left(\hat{A}, \hat{V}_{\infty}\right)<\hat{\tau}_{\infty} \leqslant \hat{\tau}_{\infty}^{+}\left(\hat{A}, \hat{V}_{\infty}\right) .
$$

It is easy to show that the upper bound must be

$$
\hat{\tau}_{\infty}^{+}\left(\hat{A}, \hat{V}_{\curvearrowright}\right)=(1-\hat{A}) \ln \hat{V}_{\infty} .
$$

Indeed, this value is the threshold for slip initiation on the virgin fault. Hence, if the remote applied stress exceeds it, there cannot exist a slip pulse with finite duration. Moreover, the smaller the difference 


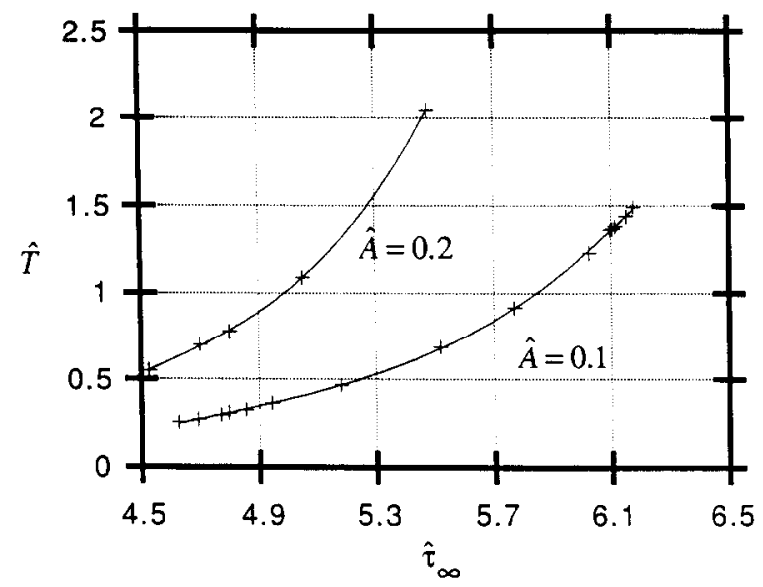

Fig. 3. Evolution of dimensionless pulse duration $\hat{T}$ as a function of dimensionless remote applied stress $\hat{\tau}_{x}$ for $\hat{V}_{x}=1000$; cases $\hat{A}=0.1$ and $\hat{A}=0.2$ are shown.

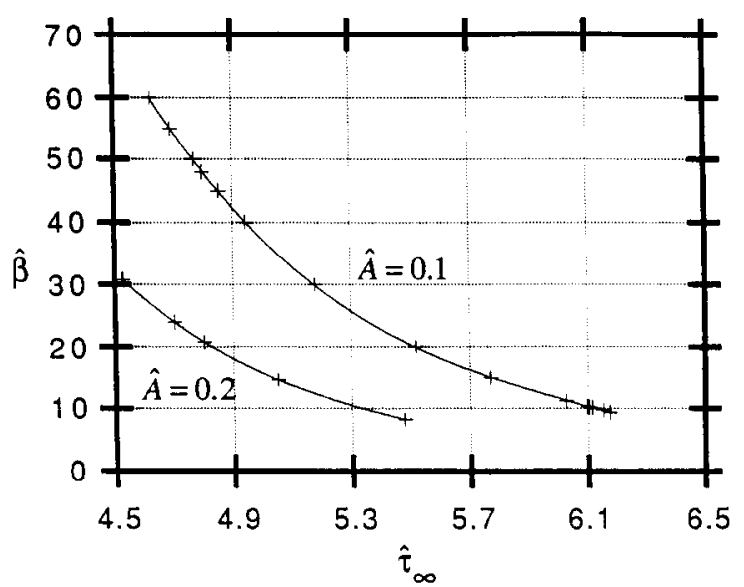

Fig. 4. Evolution of dimensionless coefficient $\hat{\beta}$ as a function of dimensionless remote applied stress $\hat{t}$, for $\hat{V}_{\text {, }}=1000 ;$ cases $\hat{A}=0.1$ and $\hat{A}=0.2$ are shown.

$$
(1-\hat{A}) \ln \hat{V}_{\infty}-\hat{\tau}_{x_{\infty}},
$$

is from the upper bound, the smaller the stress build-up precursor to the slip initiation must be. Now, the latter is given by

$$
\frac{1}{\hat{\beta}} \int_{0}^{1} \frac{\hat{V}(u)}{u} \mathrm{~d} u,
$$

which indicates that $\hat{V}(\hat{t})$ should scale with quantity (22). Our numerical computations show that both $\hat{T}$ and $\hat{\beta}$ converge to a finite value when (22) approaches 0 . This remark allows us to linearize without difficulty $(\hat{3})$ and prove that 


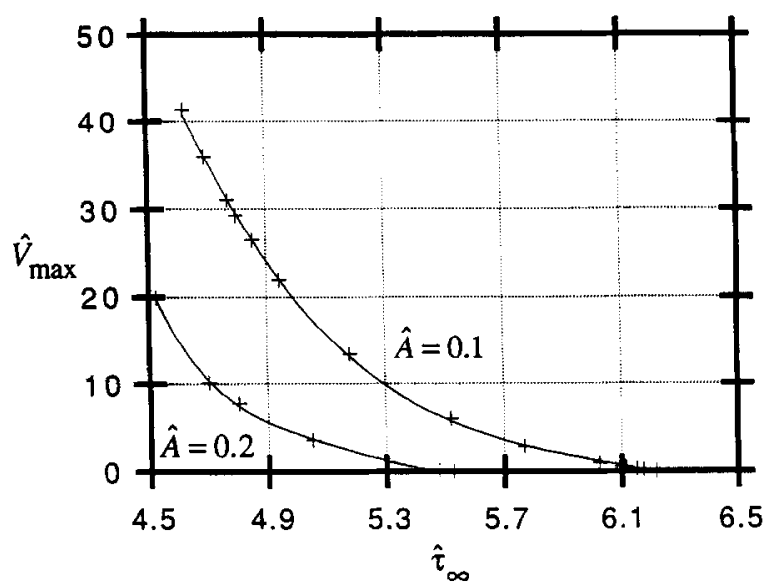

Fig. 5. Evolution of maximal dimensionless slip velocity $\hat{V}_{\max }$ as a function of dimensionless remote applied stress $\hat{\tau}_{x}$ for $\hat{V}_{\infty}=1000$; cases $\hat{A}=0.1$ and $\hat{A}=0.2$ are shown.

$$
\hat{V}(\hat{t})=\left[(1-\hat{A}) \ln \hat{V}_{\infty}-\hat{\tau}_{\infty}\right] \hat{W}(\hat{t})+o\left[(1-\hat{A}) \ln \hat{V}_{\infty}-\hat{\tau}_{\infty}\right]
$$

where $\hat{W}$ is a solution, positive on the open interval ]0,1[ and null outside, of

$$
\begin{aligned}
\frac{1}{\hat{\beta}} \mathrm{PV} \int_{0}^{1} \frac{\hat{W}(u)}{u-\hat{t}} \mathrm{~d} u+\left(1-1 / \hat{V}_{\infty}\right)\left[-\hat{A} \hat{W}(\hat{t})+\hat{T} \int_{0}^{1} \hat{W}(u) \mathrm{e}^{\hat{T}(\hat{t}-u)} \mathrm{d} u\right] \\
\text { is }\left\{\begin{array}{l}
<1 \text { when } \hat{t}<0, \\
=1 \text { when } 0 \leqslant \hat{t} \leqslant 1, \\
<1 \text { when } \hat{t}>1 .
\end{array}\right.
\end{aligned}
$$

Our numerical findings support the idea that this system has a solution only for one precise value of $(\hat{T}, \hat{\beta})$ which depends only on $\hat{A}$ and $\hat{V}_{\infty} ;$ for $\hat{V}_{\infty}=1000$, extrapolation of our results predict $(1.57,9.12)$ in the case $\hat{A}=0.1$, and $(2.4,7.6)$ in the case $\hat{A}=0.2$.

Discussion of $\hat{\tau}_{\infty}$ approaching the lower bound $\hat{\tau}_{\infty}^{-}\left(\hat{A}, \hat{V}_{\infty}\right)$ is more difficult. Our computations seem to reveal that $\hat{T}$ tends to zero, and that $\hat{\beta}, \hat{V}_{\max }$ and $\hat{\delta}_{\text {total }}$ tend to infinity corresponding to quick pulses with large offset. However, the maximal dimensionless slip velocity used was 41.4 , which is much less than the high velocity cut-off. Unfortunately, it seems difficult to consider any limit problem because the product $\hat{T}(1+\hat{V}(\hat{t}))$ which determines the rate of evolution of the state variable changes by an order of magnitude over the pulse duration. Further work is hence necessary on the limit case in which $\hat{\tau}_{\infty}$ approaches the lower bound $\hat{\tau}_{\infty}^{-}\left(\hat{A}, \hat{V}_{\infty}\right)$.

We emphasize that the non-dimensional steady pulse solutions constructed here, for given values of $\hat{A}$ and $\hat{V}_{\infty}$, and for remote loading $\hat{\tau}_{\infty}$ in a range which allows their existence, are valid for any value of the dimensionless parameter $\mu V_{0} / B c$. That parameter determines the actual, dimensional speed $V_{\text {rupture }}$ of rupture propagation. Using the identification of $\hat{\beta}$ above, where $\hat{\beta}$ values for given loadings are shown in the Tables 1 and 2 and Fig. 4, the dimensional speed is

$$
V_{\text {rupture }} \equiv \beta c=(\hat{\beta} / 2 \pi)\left(\mu V_{0} / B\right) / \sqrt{1+(\hat{\beta} / 2 \pi)^{2}\left(\mu V_{0} / B c\right)^{2}} .
$$


This has $c$ as an upper limit, but for sufficiently small $\mu V_{0} / B c, V_{\text {rupture }}$ becomes negligible compared to $c$ and the fact that our analysis framework is elastodynamic becomes irrelevant. That is, the pulse solutions derived exist also as purely quasistatic solutions of the elastic equations, an interpretation that is physically justified when $\mu V_{0} / B c$ is small enough. However, we will see in the next section that the value of $\mu V_{0} / B c$ will control whether a self-healing pulse, or instead an enlarging-crack, mode of rupture propagation actually results in transient analyses of slippage produced by sudden over-stressing of a region of fault surface; we will see that the pulse solutions associated with sufficiently small values of $\mu V_{0} / B c$ appear to be unstable.

\section{TRANSIENT ANTIPLANE ELASTODYNAMICS ANALYSIS : SPONTANEOUS RUPTURE PROPAGATION}

\subsection{Transient elastodynamic relation between stress and slip distributions}

Let $\delta(x, t)$ and $\tau(x, t)$ be the antiplane slip and stress histories along the plane $y=0$ separating two elastic half-spaces. The equations of elastodynamics require that these histories be related by an expression in the form (e.g. Cochard and Madariaga, 1994)

$$
\tau(x, t)=\tau^{0}(x, t)+f(x, t)-(\mu / 2 c) \delta,(x, t) .
$$

Here $f(x, t)$ is a linear functional of prior slip $\delta\left(x^{\prime}, t^{\prime}\right)$ over all $x^{\prime}, t^{\prime}$ satisfying $c\left(t-t^{\prime}\right)>\left|x-x^{\prime}\right|$, and $\tau^{0}(x, t)$ is the loading stress, i.e. the stress history that would have been created by external loading (e.g. as incoming waves or as a dipole layer of body force along the plane $y=0$ ) if the interface had been constrained against slip. The highest order time derivative appears in the term $(\mu / 2 c) \delta_{, t}$ representing "radiation damping" (e.g. Rice, 1993). The relation of $f(x, t)$ to $\delta\left(x^{\prime}, t^{\prime}\right)$ can be expressed as a double convolution integral given by equation (8) of Cochard and Madariaga (1994), but is simple to express, and convenient for the numerical method we present, in the spectral domain.

To evaluate the relationship in that domain, consider a full space at rest for $t<0$, and with $\tau^{0}(x, t)=0$ for all $t$, but with the interface forced to slip for $t>0$ according to

$$
u\left(x, y=0^{+}, t\right)-u\left(x, y=0^{-}, t\right) \equiv \delta(x, t)=D(t) \mathrm{e}^{\mathrm{i} k x}
$$

Here $k$ is an arbitrary wave number and $D(t)$ is an arbitrary continuous function with $D(t)=0$ for $t<0$. We seek the solution of the wave equation (A.2) for which $u_{y}(x, y, t)$ is continuous at $y=0$, where it corresponds to $\tau(x, t) / \mu$, i.e. to the stress history which would have to act to be consistent elastodynamically with the imposed slip. Writing $u(x, y, t)=U(y, t) \mathrm{e}^{i k x}$ and defining

$$
\hat{U}(y, s)=\int_{0}^{\infty} \mathrm{e}^{-s t} U(y, t) \mathrm{d} t
$$

as the Laplace transform on time, one satisfies (A.2) if 


$$
-k^{2} \hat{U}(y, s)+\hat{U}_{, y y}(y, s)=\left(s^{2} / c^{2}\right) \hat{U}(y, s),
$$

for which the solution must meet $\hat{U} \rightarrow 0$ as $|y| \rightarrow \infty$. That solution is

$$
\hat{U}(y, s)=\operatorname{sign}(y)[\hat{D}(s) / 2] \exp \left(-|y| \sqrt{k^{2}+s^{2} / c^{2}}\right) .
$$

From this we find the Laplace transform of the stress that would have to act on the interface as

$$
\hat{\tau}(x, s)=\mu u_{, y}(x, 0, t)=\mu \hat{U}_{, y}(0, s) \mathrm{e}^{\mathrm{i} k x}=-[\mu \hat{D}(s) / 2] \sqrt{k^{2}+s^{2} / c^{2}} \mathrm{e}^{\mathrm{i} k x}
$$

Since the functional $f(x, t)=\tau(x, t)+(\mu / 2 c) \delta_{t, t}(x, t)$ in these circumstances, we may write $f(x, t)=F(t) \mathrm{e}^{\mathrm{i} k x}$ where $F(t)$ is related to $D(t)$ in the Laplace domain by

$$
\hat{F}(s)=-(\mu|k| / 2) \hat{M}(s) \hat{D}(s) \quad \text { where } \hat{M}(s)=\sqrt{1+s^{2} / k^{2} c^{2}}-s /|k| c .
$$

Since $s \hat{M}(s)$ is bounded as $s \rightarrow+\infty$, there is a bounded function $M(t)$ whose transform is $\hat{M}(s)$, and the convolution theorem allows us to write

$$
F(t)=-(\mu|k| / 2) \int_{0}^{t} M(\theta) D(t-\theta) \mathrm{d} \theta,
$$

To find $M(t)$ we extend $\hat{M}(s)$ to the complex plane with branch cut on the imaginary axis between $s=-\mathrm{i}|k| c$ and $s=\mathrm{i}|k| c$, and then use the Bromwich inversion formula to write

$$
M(t)=\frac{1}{2 i \pi} \oint_{\Gamma}\left(\sqrt{1+z^{2} / k^{2} c^{2}}-z /|k| c\right) \mathrm{e}^{z t} \mathrm{~d} z
$$

where the contour $\Gamma$ can be distorted to circle once around, and shrink onto, the branch cut. Thus letting $z=\mathrm{i}|k| c \sin \psi$, with $\psi$ varying from 0 to $2 \pi$,

$$
\begin{aligned}
M(t) & =\frac{|k| c \mid}{2 \pi} \int_{0}^{2 \pi}(\cos \psi-\mathrm{i} \sin \psi) \exp (\mathrm{i}|k| c t \sin \psi) \cos \psi \mathrm{d} \psi \\
& =\frac{|k| c}{2}\left[\frac{1}{\pi} \int_{0}^{\pi} \cos (|k| c t \sin \psi) \mathrm{d} \psi+\frac{1}{\pi} \int_{0}^{\pi} \cos (|k| c t \sin \psi-2 \psi) \mathrm{d} \psi\right]
\end{aligned}
$$

The two terms within the brackets are just the well known integral representations of the Bessel functions $J_{0}$ and $J_{2}$, respectively, so that

$$
M(t)=(|k| c / 2)\left[J_{0}(|k| c t)+J_{2}(|k| c t)\right]=J_{1}(|k| c t) / t .
$$

Thus (25) above for $F(t)$, when evaluated with this convolution kernel $M(t)$, gives the functional $f(x, t)$ of (23), in response to slip history $\delta(x, t)=D(t) \mathrm{e}^{\mathrm{i} k x}$ of $(24)$, as $f(x, t)=F(t) \mathrm{e}^{\mathrm{i} k x}$.

\subsection{Numerical procedure for spontaneous slip rupture}

For our numerical method, we choose some length $\lambda$ along the $x$ axis and represent the slip $\delta$ and the functional $f$ as the Fourier series 


$$
\delta(x, t)=\sum_{n=-N / 2}^{N / 2} D_{n}(t) \mathrm{e}^{2 \pi \mathrm{in} x / \lambda}, \quad f(x, t)=\sum_{n=-N / 2}^{N / 2} F_{n}(t) \mathrm{e}^{2 \pi i n x / \lambda}
$$

where $N$ is some large even number. Here $D_{0}$ and $D_{N / 2}$ are real, and $D_{-n}$ is the complex conjugate of $D_{n}$ so that the set $\left\{D_{n}(t)\right\}$ involves $N$ real functions, and similar remarks apply to the set $\left\{F_{n}(t)\right\}$. This repeats the slip distribution, and hence the entire elastodynamic rupture process to be modeled, periodically in $x$ with interval $\lambda . N$ is chosen as a power of 2 and the Fast Fourier Transform (FFT) technique is used to rapidly relate the $\left\{D_{n}(t)\right\}$ and $\left\{F_{n}(t)\right\}$ to the values of $\delta(x, t)$ and $f(x, t)$ at $N$ sample points $x=x_{j} \equiv j h$, where $j=0,1,2, \ldots, N-1$ and $h=\lambda / N$ is the sample point spacing. Thus, to convert the histories $\delta(j h, t)$ at the $N$ sample points to histories of $f(j h, t)$, we first FFT the set $\{\delta(j h, t)\}$ to get

$$
\hat{D}_{n}(t)=\sum_{j=0}^{N-1} \delta(j h, t) \mathrm{e}^{-2 \pi i m / N}
$$

this coefficient set $\left\{\hat{D}_{n}(t)\right\}$ is related to $\left\{D_{n}(t)\right\}$ by $D_{n}=\hat{D}_{n} / N$ for $n=0$ to $N / 2-1$, $D_{N / 2}=\hat{D}_{N / 2} / 2 \mathrm{~N}$, and $D_{n}=\hat{D}_{N+n} / N$ for $n=-N / 2+1$ to -1 . Next, we calculate the $\left\{F_{n}(t)\right\}$ by the convolutions, from $(25,26)$ above with $k=2 \pi n / \lambda$,

$$
F_{n}(t)=-(\pi \mu|n| / \lambda) \int_{0}^{t} J_{1}(2 \pi|n| c \theta / \lambda) \theta^{-1} D_{n}(t-\theta) \mathrm{d} \theta .
$$

Finally we rearrange the $\left\{F_{n}(t)\right\}$ into a new set $\left\{\hat{F}_{n}(t)\right\}$, following the same rules as for rearrangement from $\left\{\hat{D}_{n}(t)\right\}$ to $\left\{D_{n}(t)\right\}$ above, and use the inverse FFT to evaluate $f(j h, t)$ as

$$
f(j h, t)=\frac{1}{N} \sum_{n=0}^{N-1} \hat{F}_{n}(t) \mathrm{e}^{+2 \pi i n / N}
$$

[In some cases it may be convenient to explicitly extract the elastostatic term in $F_{n}(t)$ :

$$
F_{n}(t)=-(\pi \mu|n| / \lambda) D_{n}(t)+(\pi \mu|n| / \lambda) \int_{0}^{t}\left[\int_{2 \pi|n| c \mid \theta ; \lambda}^{\infty} J_{1}(q) q^{-1} \mathrm{~d} q\right] \dot{D}_{n}(t-\theta) \mathrm{d} \theta
$$

this is obtained from integration by parts, noting that the integral within the brackets gives unity when $\theta=0$. A "quasistatic" approximation used by Rice (1993), perhaps better called "quasidynamic", is equivalent to retaining only the first, static elastic, term in (27), but still retaining the radiation damping term in (23) so that the formulation is capable of dealing with frictional instabilities during which a conventional quasistatic solution would not exist. That approximation effectively amounts to instantaneously propagating the final static stress changes, associated with slip, along the frictional surface, whereas the convolution term of (27) which is then neglected incorporates the proper wave-mediated radiation of those stress changes. For simulations that span many unstable events, with long intervals between them for which no dynamic effects occur, like in the modeling of sequences of crustal 
earthquakes (Rice, 1993), it may ultimately be possible to devise a numerical method that does just retain the static term of (27) during most of the history but calls on the convolution integral of (27) too as instabilities approach, and during unstable slip.]

Our numerical procedure thus consists of requiring that the $\tau$ and $\delta$ histories at the $N$ FFT sample points in $x$ be consistent with elastodynamics, i.e. satisfy (23) with $f(j h, t)$ related to the prior $\delta(j h, t)$ history as just described, and that the $\tau$ and $\delta$ histories at those sample points satisfy the friction law.

The procedure is explicit in time. Suppose $\delta$ (and hence $f$ ) and $\theta$ are known at times up to the current time $t$ at the FFT sample points and, of course, that the "loading" $\tau^{0}$ is given at time $t$ at those points. In the notation of Section 1 , (6) and (7) with a single state variable, let the constitutive law be $\tau_{\max }=F(V, \theta)$ with $\partial \theta / \partial t=\phi(V, \theta)$. Then we solve for $V$ at each of those sample points by setting

$$
F(V, \theta)+(\mu / 2 c) V=\tau^{0}+f .
$$

The left side is monotonic in $V$, by (5), so that there will be a unique solution $V>0$ when $\tau^{0}+f>F(0, \theta)$, whereas the solution is $V=0$ when the inequality is violated. We let the $V$ distribution at the sample points prevail over time step $\Delta t$, updating each $\delta$ by $V \Delta t$, using the above FFT-based procedure to update each $f$, and update $\theta$ by integrating the expression for $\partial \theta / \partial t$ over $\Delta t$ with $V$ fixed. Then we solve (28) again for $V$ at each FFT sample point and repeat the process. This procedure, with performance of the elastodynamic convolution on time in the spectral domain and use of FFT in each time step to go back and forth from real space, where the constitutive law is enforced, is closely analogous to the numerical procedure of Rice et al. (1994) for perturbed dynamic crack growth.

\subsection{Numerical results for friction law of Section 3}

We have used the friction law of Section 3, the same used in the steady pulse analysis, as the basis for several transient analyses of spontaneous rupture. The time step is chosen as $\Delta t=0.25 \mathrm{~h} / \mathrm{c}$ in examples to be shown. In addition, to properly deal numerically with the friction law we must simultaneously assure that the slip in any time step remains modest compared to $L$. Since a representative unstable slip velocity is expected to be of the order of a few times $(B-A) c / \mu$ [e.g. set the radiation damping term in (23) equal to a sudden stress drop, expected from the friction law to be of order of a few times $(B-A)]$, this means that $(B-A) c \Delta t / \mu \ll L$ or, since $\Delta t$ is order $h / c$, that $h \ll \mu L /(B-A)$. In fact, this last condition is essentially coincident with a criterion for numerical accuracy formulated by Rice (1993), who noted that the spatial resolution $h$ of his numerical grid had to be small compared to the coherent slip size, or nucleation patch size, $h^{*}$ associated with the friction law and obtained from quasistatic analysis of stability of slip (Ruina, 1983; Rice and Ruina, 1983); $h^{*}$ is the cell size $h$ at which the single-cell stiffness, $2 \mu / \pi h$ for Rice's (1993) method of discretization, is equal to the critical stiffness for stability of steady sliding. He showed that $h^{*}=2 \mu L / \pi(B-A)$ for the Ruina-Dieterich-type law that he used; the same formula applies approximately for $h^{*}$ with the friction law of Section 3 used here, at least at slip rates which are intermediate between our low and high speed limits, $V_{0}$ and $V_{\infty}$. Here, to meet $h \ll h^{*}$, we have chosen parameters so that $h=h^{*} / 24$, which 
seems to be small enough to make the actual size $h$ of the discretization essentially irrelevant to the results.

To perform the convolutions, the coefficient set $\left\{D_{n}(t)\right\}$ for all modes was stored and the kernel set $\left\{M_{n}(\theta)\right\} \equiv\left\{J_{1}(2 \pi|n| c \theta / \lambda) / \theta\right\}$ calculated for all previous time steps. We first evaluated pre-integrated kernels $\left\{\bar{M}_{n}\left(\theta_{j}\right)\right\}$, defined so that $\left\{\bar{M}_{n}\left(\theta_{j}\right)\right\} \Delta t$ is the 11-point Simpson integration of each $M_{n}(\theta)$ over $\theta_{j}$ to $\theta_{j}+\Delta t$, where $\theta_{j}=j \Delta t$ and $j=0,1,2, \ldots, t / \Delta t-1$. Then the convolution integrals were evaluated as sums of $\bar{M}_{n}\left(\theta_{j}\right) D_{n}\left(t-\theta_{j}\right) \Delta t$ over that range of $j$. [Subsequent studies (Geubelle and Morrissey, private communications, 1994) showed that a simpler 2-point trapezoidal sum, $\bar{M}_{n}\left(\theta_{j}\right)=\left[M_{n}\left(\theta_{j}\right)+M_{n}\left(\theta_{j}+\Delta t\right)\right] / 2$ is somewhat preferable not just for efficiency but also for numerical stability, particularly when our scheme is extended to tensile crack problems.] Consider the mode with shortest wavelength, for which $|n|=N / 2$. The argument $2 \pi|n| c \theta / \lambda$ of $J_{1}$ becomes $\pi \theta /(h / c)$ so that if we choose our time steps as $\Delta t=\eta h / c$ with some small $\eta$, the argument will change by $\pi \Delta t /(h / c)=\eta \pi$. In our examples to be shown we chose $\eta=0.25$, which corresponds to eight $\Delta t$-intervals within the shortest period of $J_{1}$ encountered. On the other hand, for the modes with $|n|=1$, we have totally $4 N \Delta t$-intervals per period. While there are fewer intervals per period for modes associated with the shorter wavelengths, the errors introduced in this way are made less significant by the fact that the amplitude of $J_{1}(q)$ decays as $q^{-1,2}$ so that the shorter wavelengths dampen more rapidly. We tested our algorithm with $\eta=0.25$ against analytical solutions for cracks under step-loading of their faces and the results corresponded well. The smooth, oscillation-free nature of our results shown here also suggests that the procedures with $\eta=0.25$ are suitable. In some cases $\eta=0.5$ can be used with good results. An option for future exploration is to choose the integration points more sparsely for the longer wavelength modes. Also, it should be pointed out that by working in the spectral domain the convolutions are evaluated separately for each Fourier mode, rather than as a matrix convolution in the spatial domain. This feature is very useful, especially in combination with the FFT, and makes the computations suitable for massively parallel computer architectures: we used Connection Machine 5 computers.

Results discussed here are for $\hat{A} \equiv A / B=0.2$ and $\hat{V}_{x} \equiv V_{x} / V_{0}=1000$. We take $N=2048$, i.e. $\lambda=2048 h$. As noted above, we choose $L$ and numerical FFT sample spacing $h$ to make $h=h^{*} / 24$ (and hence $\lambda=85.3 h^{*}$ ). That leaves a single free constitutive parameter $\mu V_{0} / B C$ on which solutions may depend, the parameter being the ratio of $V_{0}$ to a characteristic dynamic slip velocity $(c B / \mu)$ in a continuum sustaining stress reduction of order $B$. We consider a fault everywhere in initial state $\theta=L / V_{0}$, as it would be after a long time at rest, and imagine that a uniform applied stress $\tau^{0}$, slightly below the static strength threshold $\tau_{\max }$, acts for $t<0$ everywhere along a central section of the fault spanning the length $1024 h\left(=42.7 h^{*}\right)$. We keep $\tau^{0}$ very much lower outside that central section, so that the borders of the section act as barriers to rupture, allowing us also to study the arrest process. In subsequent plots, Figs 6(a)-9(a), we show stress measured relative to $\tau_{\mathrm{ss}, V \rightarrow x}$, the steady state value of $\tau_{\max }$ at arbitrarily high slip rate, which is just $\tau_{0}$ of (15). The threshold strength $\tau_{\max }$ before rupture is nucleated is $5.526 B$ larger than $\tau_{\mathrm{ss}, V \rightarrow \infty}$, and the figures show $\left(\tau-\tau_{\mathrm{ss} . V \rightarrow \infty}\right) /$ $5.526 B$, which corresponds to $\hat{\tau}_{x} / 5.526$. The value of $\tau^{o}(x, t<0)$ in the highly stressed central section is taken as $0.7262 B$ smaller than the threshold strength before 
rupture. This corresponds to the case of non-dimensional stress $\hat{\tau}_{\infty}=4.8$ in Table 2 and Figs 3-5, looking at curves for $\hat{A}=0.2$, and is evidently a case for which a steady traveling wave pulse solution exists. (We have confirmed that our transient numerical formulation can indeed reproduce a steady traveling wave pulse solution by considering a uniformly stressed fault and pre-conditioning by specifying the slip versus time history of a known steady pulse solution at FFT sample points within a small section of the fault, then allowing rupture to develop spontaneously on the rest of the fault. The transient solution closely reproduced the steady pulse solution up to times when interference effects from the periodic repetition of the fault intervened.)

However, in the transient solutions shown here we nucleate rupture by a step increase of the loading stress $\tau^{0}(x, t)$ at $t=0$ to make $\tau^{0}(x, t>0)$ in excess of $\tau_{\max }$ over a small section of the $x$ axis of size $80 h\left(3.4 h^{*}\right)$ within the highly stressed central section. This sudden perturbation is shown for four cases in Figs 6(a)-9(a), where we have chosen $\mu V_{0} / B c=0.50$ for Fig. $6, \mu V_{0} / B c=0.23$ for Fig. 7, and $\mu V_{0} / B c=0.10$ for Figs 8 and 9. Figures 6(a)-9(a) also show the stress in that central section following arrest of rupture propagation at the barrier ends of the section; that corresponds to the stress a few $\Delta t$ after all slip has ceased, and will be modestly different from the
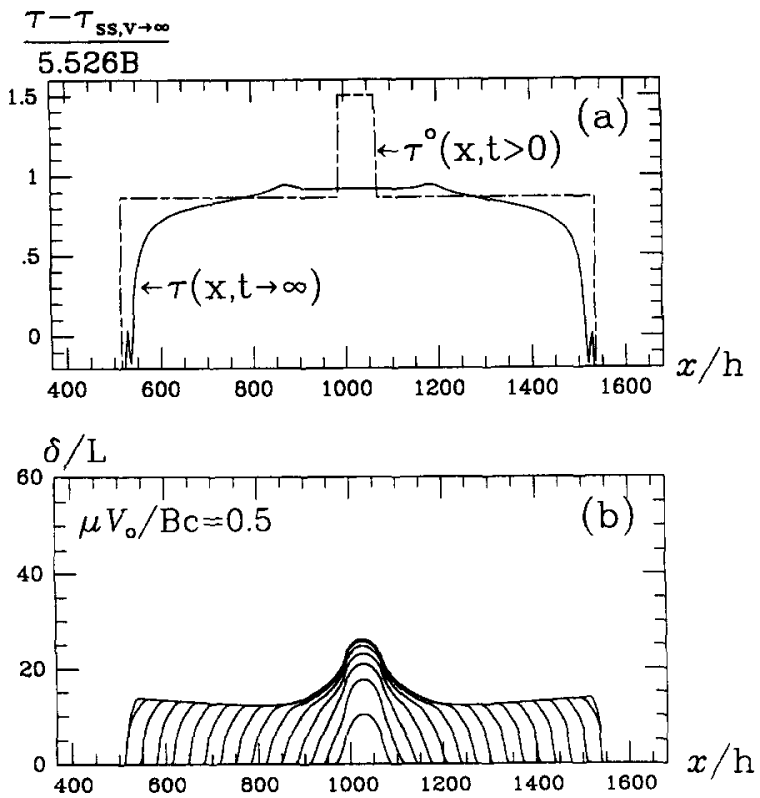

Fig. 6. Transient rupture propagation results for $\hat{A}=0.2, \hat{V}_{x}=1000$ and $\mu V_{0} / B c=0.50$. (a) Stress $\tau$ measured relative to $\tau_{s s, v \rightarrow \infty}$, the steady state value of $\tau_{\max }$ at arbitrarily high slip rates; the threshold strength $\tau_{\text {max }}$ before rupture is nucleated is $5.526 B$ larger than $\tau_{\mathrm{ss}, V^{\prime} \rightarrow \infty}$. Initial stressing $\tau^{0}(x, t<0)$ is high and uniform over a central section spanned by 1024 FFT sample points, of spacing $h=h^{*} / 24$, between $x=512 h$ and $1536 h$, where the periodic repeat distance $\lambda=2048 h=85.3 h^{*}$. A perturbation of $\tau^{0}$ is applied over a section of length $80 h$ at $t=0$ to nucleate rupture, so that the loading stress $\tau^{\circ}(x, t>0)$ corresponds to the rectangular distribution shown, with $\tau^{\circ}$ reduced far below $\tau_{\mathrm{ss}, V \rightarrow \infty}$ outside the central section to form barriers to rupture. The final stress distribution over that central section, once rupture has ceased, is also shown. (b) Slip versus distance during rupture propagation over the central section, with curves drawn at time intervals $140 \Delta t=35 \mathrm{~h} / \mathrm{c}$. In this case rupture propagates as a self-healing slip pulse but the pulse is not a steady traveling wave. 

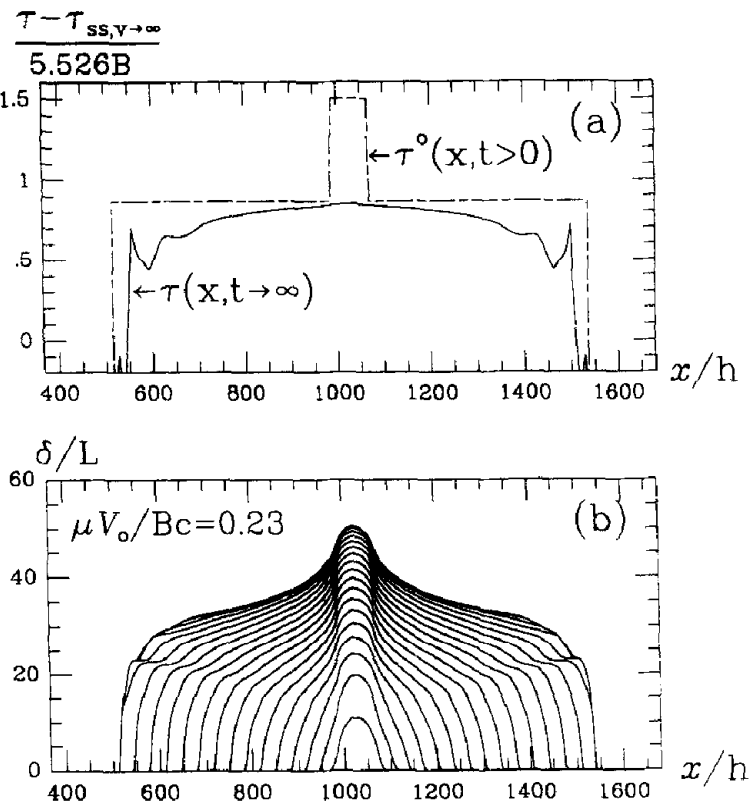

Fig. 7. Samc as for Fig. 6, but with $\mu V_{0} / B c=0.23$. This $\mu V_{0} / B c$ is in the range of transition from the selfhealing pulse mode of propagation like in Fig. 6 to the enlarging-shear-crack mode as in Fig. 8.
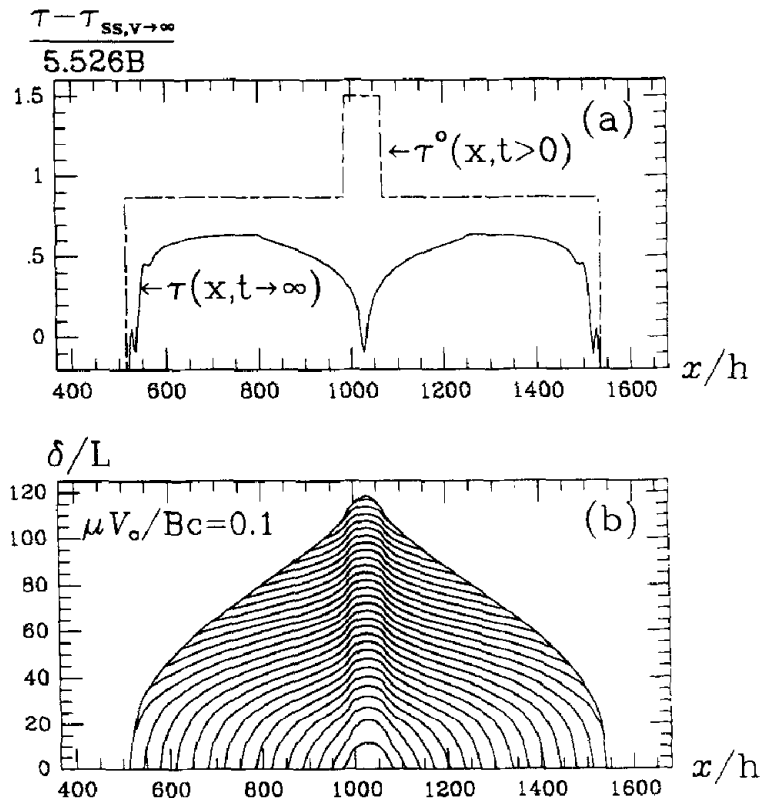

Fig. 8. Same as for Figs 6 and 7 , but with $\mu V_{0} / B c=0.10$. In this case rupture propagates in a mode like that of a classical enlarging-shear-crack. 

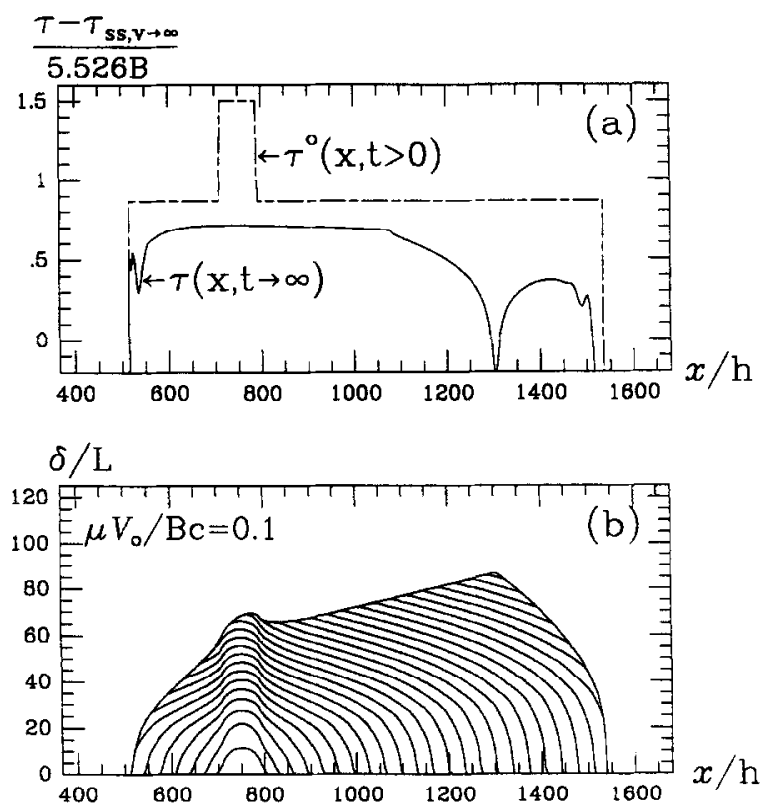

Fig. 9. Same as for Figs $6-8$, but with $\mu V_{0} / B c=0.10$ and now with the loading perturbation applied towards the left border of the highly stressed central section. The conditions favor the enlarging-shearcrack modc of propagation but effects of arrest at the left barricr propagate towards the rightward moving

front of the rupture and create a self-healing pulse by the mechanism discussed by Johnson (1990).

final static stress distribution. The slip histories over the central section are shown in Figs 6(b)-9(b), where the curves are separated by uniform time increments of $140 \Delta t=35 h / c$.

We observe the following from the results in Figs 6 9:

(1) Although the constitutive parameters and loading over the central section are consistent with a steady traveling wave pulse, that solution is not observed when ruptures are started by suddenly over-stressing a small region as described. Thus the steady traveling wave pulse solution is either unstable (but not violently so because, as noted, we can reproduce that solution with specially chosen preconditioning) or, in the language of dynamical systems, the solution has a limited basin of attraction.

(2) Examining Figs 6(b)-8(b), which correspond to identical loading but decreasing values of $\mu V_{0} / B c$, we see that there is a transition in the mode of rupture propagation. Relatively high values of $\mu V_{0} / B c$, like 0.50 as in Fig. 6, lead to a self-healing pulse mode of propagation, although it is not steady but grows (and, with other loading conditions, may decay) with propagation distance. But low values of $\mu V_{0} / B c$, like 0.10 as in Fig. 8, lead to an entirely different mode of propagation, like that of a classical enlarging shear crack. Figure 7 shows a case in the vicinity of the transition between these two modes, and is for $\mu V_{0} / B C=0.23$. Thus we have a transition, at this loading level, from a self-healing slip pulse mode for $\mu V_{0} / B c$ greater than approximately $0.23-0.26$, to a crack-like mode for $\mu V_{0} / B c$ less than approximately $0.23-0.26$. The transition range does depend on the loading level, and another series 
of calculations that we did (results not shown) at a higher loading level, corresponding to the case $\hat{\tau}_{\infty}=5.40$ in Table 2, gave the transition from a self-healing pulse to a crack-like rupture mode around $\mu V_{0} / B c=0.50$. Similar transitions, dependent on a characteristic slip rate for velocity weakening, were reported by Tullis et al. (1992) and Beeler and Tullis (1995). Using the values of $\hat{\beta}$ in Table 2 for the two values of $\hat{\tau}_{x}$ considered in our transient analyses, and using the expression for $V_{\text {rupture }}$ at the end of Section 5, we find in both cases that the crack-like mode of rupture propagation is observed when the propagation speed for the steady, traveling wave pulse solution falls below about $0.6 c$.

(3) Figures (8) and (9) are for the same value $\mu V_{0} / B c=0.10$, and same magnitude of loading perturbation, but that perturbation is applied at the middle of the central, highly stressed, section in Fig. 8 and towards a border of that section in Fig. 9. We see that arrest effects from the barrier at the left in Fig. 9 then chase the rightward running front of the rupture, thus also producing a short-duration slip pulse (although not so short as in Fig. 6), even though the conditions would otherwise favor the cracklike rupture mode. This provides an example of the mechanism of self-healing pulse generation discussed by Johnson (1990).

(4) Extremely non-uniform stress distributions are left after the end of rupture. A large positive concentration of stress would also show outside the barriers, had our assignment of $\tau^{0}$ to values very much lower than $\tau_{\mathrm{ss} . V \rightarrow \infty}$ outside the central section not suppressed it. An important issue for future work is to understand whether such strong non-uniformity would still result with less abrupt ways of starting and stopping the rupture, e.g. by gradual development of an initially quasistatic frictional instability, and by rupture arrest due to smooth variation of rheological properties towards stable velocity strengthening with increase of crustal depth, like in the modeling of repetitive earthquakes by Rice (1993). A further issue of importance to seismological theory is that of whether such inertial dynamics-based heterogeneity of stress on essentially smooth faults is sufficient to sustain spatio-temporal complexity of seismic response, including observed frequency-magnitude statistics of smaller earthquakes, or whether appeal to geometric disorder to provide local rupture-stopping barriers, like fault bends or offsets, is necessary. See Rice (1993), Cochard and Madariaga (1994), Shaw (1994), Myers et al. (1994), and Ben-Zion and Rice (1995) for related discussion.

\section{DISCUSSION}

We remark that at present there does not seem to exist constitutive data for any material to characterize the state-dependent part of the friction law, which we have shown to be critical to whether or not slip-pulse solutions exist on a smooth unbounded fault, over the full range of slip rates experienced in an instability. This is a critical issue, since we have shown that not all plausible forms of the constitutive law allow such pulse solutions. For example, in the case of granite, recent summaries (Kilgore et al., 1993 ; Weeks, 1993) of extensive laboratory studies give results suitable to characterize constitutive response for slip rates $V$ between $10^{-7} \mathrm{~mm} / \mathrm{s}(3 \mathrm{~mm} / \mathrm{yr})$ and $1 \mathrm{~mm} / \mathrm{s}$. These are focused on a $V$ range critical for understanding the nucleation 
of rupture but may possibly be largely irrelevant to whether pulse-like rupture propagation occurs, since average slip rates during natural earthquakes seem to be of order $10^{3} \mathrm{~mm} / \mathrm{s}$ (Heaton, 1990), and are expected to be larger near the advancing front of the rupture.

The low speed granite results can be fit, approximately, with $\hat{A} \approx 0.7, L \approx 10^{-5}$ $\mathrm{mm}, V_{\infty} \approx 0.1 \mathrm{~mm} / \mathrm{s}$, and $V_{0} \approx 10^{-7} \mathrm{~mm} / \mathrm{s}$ or smaller. This $\hat{A}$ is much larger than the two values $(0.1$ and 0.2$)$ that we have considered here. However, serpentine (Reinen et al., 1992) in a similar low speed range shows, at slip rates faster than about $10^{-3}$ $\mathrm{mm} / \mathrm{s}, \hat{A}$ values of 0.1 .

Technological materials do not seem to be similarly well characterized at present within the rate- and state-dependent constitutive framework, but Prakash and Clifton $(1992,1993)$ have recently reported results of the sliding of a hard steel and a titanium alloy on tungsten carbide that they were able to partially characterize within that framework. Further, those were obtained by a shock wave technique with oblique impact and involve slip speeds from about $2-30 \times 10^{3} \mathrm{~mm} / \mathrm{s}$, a range which would also be interesting for earthquakes. We have chosen values of our constitutive parameters here largely on the basis of computational feasibility; it is possible that the numerical difficulties would be yet more challenging if realistic constitutive parameters, once known for the entire slip range involved, were to be used.

To examine further the slip rate range $V_{0} \approx(0.25-0.50)(B c / \mu)$ reported in Section 6.3 , in which there is the transition, dependent on loading level, from the self-healing pulse to the classical enlarging-crack rupture mode, let us tentatively assume that $B$ is of order $0.015 \bar{\sigma}$, where $\bar{\sigma}$ is the effective normal stress on the sliding surface. This sort of $B$ value is consistent with the data from the low speed tests on granite and quartzite discussed above and earlier. Then, with $\mu=30 \mathrm{GPa}$ and $c=3 \mathrm{~km} / \mathrm{s}$, the transitional range is $V_{0}=23-45 \mathrm{~mm} / \mathrm{s}$ at $\bar{\sigma}=60 \mathrm{MPa}$, and scales with $\bar{\sigma}$. The obvious problem is that such values of $V_{0}$ lie outside the range of speeds for which the constitutive data, leading to the estimate of $B$, was taken. This points to the necessity of friction experiments at higher slip rates to confirm whether short duration slip pulses can be explained on the basis of frictional constitutive response. If we used only the low speed laboratory data that provided the experimental basis for the logarithmic forms in the Dieterich-Ruina class of constitutive laws, then we would conclude that $\mu V_{0} / B c$ is sufficiently small that the steady, traveling wave pulse solution represented a slow quasistatic disturbances, but one that is very likely unstable and would develop under perturbation into a crack-like dynamic rupture.

Again, we emphasize that slip-pulse solutions will not exist at all for some parameter ranges or for some classes of constitutive laws within the rate- and state-dependent framework. They do not exist at any sub-sonic propagation speed when one attempts to describe friction on the ruptured surface within the classical, purely rate-dependent, constitutive framework.

In future work it might be useful to address the pulse problem with other regularizations of the Dieterich-Ruina model, more respectful of the contact time interpretalion of the stale variable $\theta$. Indeed, we somewhat altered this interpretation in Section 3 and it might be preferred to let $\theta$ diverge towards infinity like the time spent in stationary contact. Then, for $\tau_{\max }$ to remain bounded, we have to introduce a cut-off in its $\theta$ dependency. A candidate to replace $\left(13_{1}\right)$ is : 


$$
\tau_{\max }(t)=\tau_{0}+A \ln \left\{\left[V_{0}+V(t)\right] /\left[V_{\infty}+V(t)\right]\right\}-B \ln \left\{\left[\theta_{0}+\theta(t)\right] /\left[\theta_{\infty}+\theta(t)\right]\right\} .
$$

If we keep the (state evolution) ordinary differential equation $\left(13_{2}\right)$ though, a problem arises when one considers a fault that is stuck in stationary contact for a very large (infinite) duration, so that $\theta(t)=\infty$ : when slipping starts, the differential equation would not change $\theta$ in a finite time. Now, if $\theta$ is very large instead of infinite, the statedependent part of $\tau_{\max }$ evolves very slowly. This defect can be solved by introducing a state evolution equation of the form

$$
\mathrm{d} \theta(t) / \mathrm{d} t=1-[\theta(t) V(t) / 2 L]^{2} .
$$

Even with bounded $V(t)$, the squared term allows for otherwise finite solutions $\theta(t)$ starting with initial condition $\theta(0)=\infty$. This comes from the well known finite time divergence $\theta(+\infty)=+\infty$ of the backward equation, which reads $\mathrm{d} \theta(t) / \mathrm{d} t=-1+[\theta(t) V / 2 L]^{2}$, with any initial condition $\theta$ greater than the repulsive critical value $2 L / V$. The factor 2 leaves unchanged the characteristic length $L$ of the model in the vicinity of the long term limit for stationary velocity, $\theta=2 L / V$. Further analysis based on this law is left for future work.

\section{ACKNOWLEDGEMENTS}

The study was supported by the Office of Naval Research Mechanics Program (grant N00014-90-J-1379), the U.S. Geological Survey National Earthquake Hazards Reduction Program (grant 1434-94-G-2450) and the NSF Southern California Earthquake Center (subcontract PO 621911 from the University of Southern California). G. P. also acknowledges support from the Ecole des Mines de Paris and Laboratoire de Mécanique des Solides à l'Ecole Polytechnique, France. The transient elastodynamics calculations of Section 6 were aided by grants of time on Connection Machine 5 computers, from NSF at the National Center for Supercomputer Applications at the University of Illinois, and from ARPA under auspices of Project Scout of MIT, Harvard, Boston University and Thinking Machines Inc. We are grateful to colleagues Y. Ben-Zion, P. H. Geubelle, J. Kysar and J. Morrissey for discussions concerning development of the spectral procedure for transient elastodynamics. The study was initiated during the participation of GP and JRR in the 1992 Program on Spatially Extended Nonequilibrium Systems at the NSF Institute for Theoretical Physics in Santa Barbara, and JRR acknowledges helpful discussions with M. N. Barber, J. S. Langer and C. R. Myers at that time.

\section{REFERENCES}

Andrews, D. J. (1976) Rupture velocity of plane-strain shear cracks. J. Geophys. Res. 18, 56795687.

Andrews, D. J. (1985) Dynamic plane-strain shear rupture with a slip-weakening friction law calculated by a boundary integral method. Bull. Seismol. Soc. Am. 75, 1-21.

Andrews, D. J. (1994) Dynamic growth of mixed-mode shear cracks. Bull. Seismol. Soc. Am. 84, 1184-1198.

Beeler, N. M. and Tullis, T. E. (1995) Self-healing slip pulses in dynamic rupture models due to velocity-dependent strength. Bull. Seismol. Soc. Am. (in press).

Beeler, N. M., Tullis, T. E. and Weeks, J. D. (1994) The roles of time and displacement in the evolution effect in rock friction. Geophys. Res. Lett. 21, 1987-1990. 
Ben-Zion, Y. and Rice, J. R. (1995) Slip patterns and earthquake populations along different classes of faults in elastic solids. J. Geophys. Res. 100, 12959-12983.

Boatwright, J. (1988) The seismic radiation from composite models of faulting. Bull. Seismol. Soc. Am. 78, 489-508.

Burridge, R., Conn, G. and Freund, L. B. (1979) The stability of a rapid mode II shear crack with finite cohesive traction. J. Geophys. Res. 84, 2210-2222.

Cochard, A. and Madariaga, R. (1994) Dynamic faulting under rate-dependent friction. Pure Appl. Geophys. 142, 419-445.

Day, S. M. (1982) Three-dimensional finite difference simulation of fault dynamics : rectangular faults with fixed rupture velocity. Bull. Seismol. Soc. Am. 72, 705-727.

Dieterich, J. H. (1978) Time-dependent friction and the mechanism of stick-slip. J. Geophys. Res. 84, 2161-2168.

Dieterich, J. H. (1979) Modeling of rock friction-1. Experimental results and constitutive equations. Pure Appl. Geophys. 116, 790-806.

Dieterich, J. H. (1981) Constitutive properties of faults with simulated gouge. In Mechanical Behavior of Crustal Rocks (ed. N. L. Carter, M. Friedman, J. M. Logan and D. W. Stearns), pp. 103-120, Geophysical Monograph 24. AGU, Washington.

Dieterich, J. H. (1986) A model for the nucleation of earthquake slip. In Earthquake Source Mechanics (ed. S. Das) Vol. 6 pp. 37-47, Geophysical Monograph 37. AGU, Washington.

Dieterich, J. H. (1992) Earthquake nucleation on faults with rate- and state-dependent strength. Tectonophysics 211, 115-134.

Dieterich, J. H. and Conrad, G. (1984) Effect of humidity on time- and velocity-dependent friction in rocks. J. Geophys. Res. 89, 4196-4202.

Freund, L. B. (1979) The mechanics of dynamic shear crack propagation. J. Geophys. Res. 84, 2199-2209.

Gu, J.-C., Rice, J. R. Ruina, A. L. and Tse, S. T. (1984) Slip motion and stability of a single degree of freedom elastic system with rate and state dependent friction. J. Mech. Phys. Solids 32, 167-196.

Heaton, T. H. (1990) Evidence for and implications of self-healing pulses of slip in earthquake rupture. Phys. Earth Planetary Interiors 64, 1-20.

Johnson, E. (1990) On the initiation of unidirectional slip. Geophys. J. Int. 101, 125-132.

Johnson, E. (1992) The influence of the lithospheric thickness on bilateral slip. Geophys. J. Int. 108, $151-160$.

Kilgore, B. D., Blanpied, M. L. and Dieterich, J. H. (1993) Velocity dependent friction of granite over a wide range of conditions. Geophys. Res. Lett. 20, 903-906.

Mushkelishvili, N. I. (1953) Singular Integral Equations (translation edited by J. R. M. Radok, P. Noordhoff). N. V., Groningen.

Myers, C. R., Shaw, B. E. and Langer, J. S. (1994) Slip complexity in a crustal-plane model of an earthquake fault. Preprint manuscript NSF-ITP-94-98, Inst. for Theor. Phys., Santa Barbara.

Okubo, P. G. (1989) Dynamic rupture modeling with laboratory-derived constitutive laws. $J$. Geophys. Res. 94, 12,321-12,335.

Okubo, P. and Dieterich, J. H. (1986) State variable fault constitutive relations for dynamic slip. In Earthquake Source Mechanics, (ed. S. Das), Vol. 6, pp. 25-35, Geophysical Monograph 37. AGU, Washington.

Papageorgiou, A. S. and Aki, K. (1983) A specific barrier model for the quantitative description of inhomogeneous faulting and the prediction of strong ground motion. Bull. Seismol. Soc. Am. 73, 693722.

Prakash, V. and Clifton, R. J. (1992) Pressure-shear plate impact measurement of dynamic friction for high speed machining applications. In Proceedings of VII International Congress on Experimental Mechanics, Las Vegas, June 1992, pp. 556-564. Society of Experimental Mechanics, Bethel, Conn.

Prakash, V. and Clifton, R. J. (1993) Time-resolved dynamic friction measurements in pressureshear. In Experimental Techniques in the Dynamics of Deformable Solids, Applied Mechanics Div. Vol. 165 (AMD-Vol. 165), pp. 33-48. American Society of Mechanical Engineers, New York. 
Reinen, L. A., Tullis, T. E. and Weeks, J. D. (1992) Two-mechanism model for frictional sliding of serpentine. Geophys. Res. Lett. 19, 1535-1538.

Rice, J. R. (1968) Mathematical analysis in the mechanics of fracture. In Fracture (ed. H. Liebowitz), Vol. II, pp. 191-311. Academy Press, New York.

Rice, J. R. (1993) Spatio-temporal complexity of slip on a fault. J. Geophys. Res. 98, 98859907.

Rice, J. R. and Ruina, A. L. (1983) Stability of steady frictional slipping. J. Appl. Mech. 50, 343-349.

Rice, J. R., Ben-Zion, Y. and Kim, K. S. (1994) Three-dimensional perturbation solution for a dynamic planar crack moving unsteadily in a model elastic solid. J. Mech. Phys. Solids 42, $813-843$.

Ruina, A. L. (1980) Friction laws and instabilities : a quasistatic analysis of some dry frictional behavior. Ph.D. Thesis, Division of Engineering, Brown University, Providence.

Ruina, A. L. (1983) Slip instability and state variable friction laws. J. Geophys. Res. 88, 10359 10370.

Shaw, B. E. (1994) Complexity in a spatially uniform continuum fault model. Geophys. Res. Lett. 21, 1983-1886.

Tikhonov, A. N. and Goncharsky, A. V. (1987) Ill-Posed Problems in the Natural Sciences. Mir Publishers, Moscow.

Tullis, T. E. Beeler, N. M. and Weeks, J. D. (1992) Frictional healing of dynamic earthquake rupture (abstract). EOS, Trans. Amer. Geophys. Union 73(43) Suppl. AGU 1992 Fall Meeting; Abstract S41D-7, p. 388.

Weeks, J. D. (1993) Constitutive laws for high velocity frictional sliding and their influence on stress drop during unstable slip. J. Geophys. Res. 98, 17,637-17,648.

Weeks, J. D. and Tullis, T. E. (1985) Frictional sliding of dolomite : a variation in constitutive behavior. J. Geophys. Res. 90, 7821-7826.

\section{APPENDIX A : ELASTODYNAMIC REPRESENTATION FOR STEADY PULSE AS A TRAVELING WAVE}

This Appendix aims to establish relationships (1) and (2). The displacement of the upper half of the medium $\{y>0\}$ along axis $z$ is a steady traveling wave, and it can be written by means of a function of two variables $f\left(x_{1}, x_{2}\right)$ as

$$
u(x, y, t)=f\left(\frac{x-\beta c t}{\sqrt{1-\beta^{2}}}, y\right)
$$

The displacement is a solution of the elastodynamic equation :

$$
u_{x x}+u_{. r y}=\frac{1}{c^{2}} u_{. t t}
$$

Straightforward algebra then shows that $f$ is an harmonic funtion on $\{y>0\}$. Classical results on such functions then yield (see e.g. Rice, 1968)

$$
\frac{\partial f}{\partial x_{2}}\left(x_{1}, x_{2}=0^{+}\right)=\frac{\partial f}{\partial x_{2}}\left(x_{1}= \pm \infty, x_{2}=0^{+}\right)-\frac{1}{\pi} \mathrm{PV} \int_{-\infty}^{+x} \frac{\partial f}{\partial \eta}\left(\eta, x_{2}-0^{+}\right) \frac{\mathrm{d} \eta}{x_{1}-\eta} .
$$

Identifying

$$
\tau(t)=\mu \frac{\partial u}{\partial y}\left(x=0, y=0^{+}, t\right), \quad V(t)=2 \frac{\partial u}{\partial t}\left(x=0, y=0^{+}, t\right),
$$

using representation (A.1) finally yields (1) and (2). 


\section{APPENDIX B : ASYMPTOTIC SHAPE OF A PULSE NEAR ITS TIPS : \\ DERIVATION OF (8) and (9)}

\section{B.1. Case of the slip initiating tip}

We first address the problem around $t=0$. In fact, the result we are able to prove is slightly weaker, because we assume that $V(t)$ is a power law with positive (for continuity) exponent. We consider the case when the frictional state is stationary before sliding begins $($ at $t=0)$. This is reasonably consistent with the slip velocity being constant (null) for negative time, so that the state variables, would it be otherwise at some remote past time, converge to a stationary solution $\{\theta(0)\}$ of $(7)$ written for $V(t=0)=0$ :

$$
\{0\}=\{\dot{\theta}\}=\{\varphi(V=0,\{\theta(0)\})\} .
$$

Then, at first order, the state variables evolution equation (7) provides

$$
\{\dot{\theta}(t)\}=O\left(\frac{\partial\{\varphi(V=0,\{\theta(0)\})\}}{\partial V} \alpha t^{\prime}\right)
$$

[the notation $O(f(t))$ is now introduced, meaning any function $g(t)$ so that $g(t) / f(t)$ be bounded in the $t$-limit considered] and, through time integration,

$$
\{\theta(t)\}=\{\theta(0)\}+O\left(t^{\gamma+1}\right) .
$$

Expanding definition (6), we finally obtain from the frictional behavior the asymptotic expression of $\tau_{\max }$ for small $t>0$ :

$$
\tau_{\max }(t)=F(0,\{\theta(0)\})+\frac{\partial F(0,\{\theta(0)\})}{\partial V} \alpha t^{\gamma}+o\left(t^{\gamma}\right) .
$$

On the other hand, the properties of generalized function PV $(1 / x)$ proven in Appendix C allows us to derive easily, from the elastodynamic (1) and (2), the following asymptotic expressions:

$$
\left\{\begin{array}{l}
\text { case (i): } \quad 0<\gamma<1 \Rightarrow \tau^{\mathrm{IE}}(t)=\tau_{\infty}+g \int_{0}^{T} \frac{V(u)}{u} \mathrm{~d} u+\alpha g t^{\prime} \pi \tan (\pi(\gamma-1 / 2))+o\left(t^{\prime}\right), \\
\text { case (ii): } \quad \gamma=1 \Rightarrow \tau^{\mathrm{IE}}(t)=\tau_{\infty}+g \int_{0}^{r} \frac{V(u)}{u} \mathrm{~d} u+\alpha g t|\ln t|+O(t), \\
\text { case (iii): } \quad \gamma>1 \Rightarrow \tau^{\mathrm{IE}}(t)=\tau_{\infty}+g \int_{0}^{T} \frac{V(u)}{u} \mathrm{~d} u+g t \int_{0}^{T} \frac{V(u)}{u^{2}} \mathrm{~d} u+o(t) .
\end{array}\right.
$$

Notice that $V(u) / u$ is integrable at point $u=0$ because $\gamma>0$. Now, because velocity is positive for $t>0$, the equality $\tau^{\mathrm{IE}}(t)=\tau_{\max }(t)$ necessarily holds, which implies, first, that case (i) be encountered, second, that (9) be fulfilled, and third a new condition

$$
\tau_{\max }(t=0)=F(0,\{\theta(0)\})=\tau_{\infty}+g \int_{0}^{T} \frac{V(u)}{u} \mathrm{~d} u,
$$

which has the following transparent physical meaning: sliding begins when the frictional threshold is reached by the sum of the remote stress, $\tau_{\infty}$, and of the stress pulse created by the steady propagating sliding pulse.

The following remarks may he added: (i) If the stationary frictional state hypothesis (B.1) does not hold, then the case of the initiation tip would be treated in the same way as the slip arresting tip (see Section B.2 below) and the critical exponent in $\left(8_{1}\right)$ would be $\gamma+1$. (ii) In more mathematical words, we encountered an asymptotic eigenvalue problem: some fixed functional operator $A$ (here given by equations (1) and (2)) and real number $\lambda$ [here given by 
the instantaneous derivative (5)] being given, what condition(s) should any function $V(t)$, null for negative argument, fulfill which would respect

$$
((A V)(t)-(A V)(0))=\lambda V(t)+o(V(t))
$$

in the vicinity of $t=0^{+}$?

The treatment which we presented does not ensure uniqueness because we considered only the particular case when the unknown function $V(t)$ behaves like a power function near the origin.

\section{B.2. Case of the slip arresting tip. We now address the problem around $\mathrm{t}=\mathrm{T}$}

Let $\gamma^{\prime}$ be the exponent on $(T-t)$, in an expression for $V(t)$ like in $\left(8_{2}\right)$. Let us first obtain some information from considering the region $t \geqslant T$, just after the arrest. Notice that there is no reason for the state to be stationary, so that, unlike in the case of the initiating tip.

$$
\{\dot{\theta}\}=\{\varphi(V=0,\{\theta(T)\})\} \neq\{0\} .
$$

Then, expansion of (6) for $t \rightarrow T^{+}$yields

$$
\left.\tau_{\max }(t)=F(0,\{\theta(T)\})+\alpha_{1}(t-T)+O(t-T)^{2}\right), \text { with } \alpha_{1}=\frac{\partial F(0,\{\theta(T)\})}{\partial\{\theta\}}\{\dot{\theta(T)}\} .
$$

Besides, the integral in (1) is regular for $t>T$, and

$$
\frac{\mathrm{d} \tau^{\mathrm{IE}}}{\mathrm{d} t}(t)=g \int_{0}^{T} \frac{V(u)}{(u-t)^{2}} \mathrm{~d} u,
$$

which would tend towards $+\infty$ for $t \rightarrow T^{+}$if $\gamma^{\prime} \leqslant 1$. Such is not compatible with $\tau^{1 \mathrm{E}}(t) \leqslant \tau_{\max }(t)$, which proves that $\gamma^{\prime}>1$.

Let us now consider the region $t \leqslant T$ in a way similar to that used in the case on the initiation tip. On the one hand, expanding (6) yields

$$
\tau_{\max }(t)-F(0,\{0(T)\})+\alpha_{1}(t-T)+\frac{\partial F(0,\{\theta(T))\})}{\partial V} \alpha^{\prime}(T-t)^{\prime \prime}+O\left((t-T)^{2}\right)+o\left((T-t)^{\prime}\right) .
$$

with $\alpha_{1}$ as given in (11). On the other hand, the method used in Appendix $C$ can easily be adapted to the right bound of the interval, $t \rightarrow T$ (on $t<T$ ); see the remark ending that Appendix. Thus, provided that $1<\gamma^{\prime} \leqslant 2$, one gets :

$$
\left\{\begin{array}{l}
\text { case (i) : } \\
\quad 1<\gamma^{\prime}<2 \Rightarrow \tau^{\mathrm{IE}}(t)=\tau_{y}+g \int_{0}^{T} \frac{V(u)}{u-T} \mathrm{~d} u+g(t-T) \int_{0}^{T} \frac{V(u)}{(u-T)^{2}} \\
\quad+\alpha^{\prime} g(T-t)^{\gamma^{\prime}} \pi \tan \left(\pi\left(\gamma^{\prime}-3 / 2\right)\right)+\mathrm{o}\left((T-t)^{\prime}\right), \\
\text { case (ii) : } \\
\quad \gamma^{\prime}=2 \Rightarrow \tau^{\mathrm{IE}}(t)=\tau_{x}+g \int_{0}^{T} \frac{V(u)}{u-T} \mathrm{~d} u+g(t-T) \int_{0}^{T} \frac{V(u)}{(u-T)^{2}} \mathrm{~d} u \\
\quad+\alpha^{\prime} g(T-t)^{2}|\ln (T-t)|+O\left((T-t)^{2}\right) .
\end{array}\right.
$$

As in the case of slip onset, the equality $\tau^{\mathrm{IE}}(t)=\tau_{\text {max }}(t)$ forbids case (ii) and results in $\gamma^{\prime}=\gamma+1$, plus two additional conditions: 


$$
\tau_{\max }(t=T)=F(0,\{\theta(T)\})=\tau_{\infty}+g \int_{0}^{T} \frac{V(u)}{u-T} \mathrm{~d} u,
$$

with a meaning similar to that for $\tau_{\max }(t=0)$, and :

$$
\dot{\tau}_{\max }(t=T)=\frac{\partial F(0,\{\theta(T)\})}{\partial\{\theta\}}\{\varphi(0,\{\theta(T)\})\}=g \int_{0}^{T} \frac{V(u)}{(u-T)^{2}} \mathrm{~d} u,
$$

where the last integral is regular because $\gamma^{\prime}>1$.

We remark that a more detailed analysis shows that in fact, the exponent $\gamma^{\prime}$ might be any $n+\gamma, n$ being a positive integer. However, the cases with $n$ greater than or equal to 2 are regular enough not to raise numerical complications, as does the case with $n=1$. For this reason, the numerical algorithms presented in the following take explicitly the latter case into account, and are a fortiori able to treat the former ones.

\section{APPENDIX C : SUPPORTING CALCULATIONS}

This Appendix is devoted to calculating the asymptotic behavior of

$$
f(t)=\mathrm{PV} \int_{-\infty}^{+\infty} g(t+u) \frac{\mathrm{d} u}{u},
$$

for $t \rightarrow 0^{+}$, assuming that the support of $g$ is $[0, T]$, and that $g(t)=t^{\gamma} h(t)$, with $\gamma$ positive and $h(0)=1$, and $h$ being a Hölder continuous function of exponent $\varepsilon>0$; that is, there exists a constant $C$ such that

$$
\forall(x, y),|h(x)-h(y)|<C|x-y|^{\varepsilon}
$$

The result is

$$
\begin{cases}\text { case (i): } & 0<\gamma<1 \Rightarrow f(t)=\int_{0}^{T} \frac{g(u)}{u} \mathrm{~d} u+t^{\prime} \pi \tan (\pi(\gamma-1 / 2))+o\left(t^{\prime}\right), \\ \text { case (ii) : } & \gamma=1 \Rightarrow f(t)=\int_{0}^{T} \frac{g(u)}{u} \mathrm{~d} u+t|\ln t|+O(t), \\ \text { case (iii) : } & \gamma>1 \Rightarrow f(t)=\int_{0}^{T} \frac{g(u)}{u} \mathrm{~d} u+t \int_{0}^{T} \frac{g(u)}{u^{2}} \mathrm{~d} u+o(t) .\end{cases}
$$

Let us begin with cases (i) and (ii). First, we perform the change of variable $u=w-t$ in (C.1), and, noticing that

$$
\frac{1}{w-t}=\frac{1}{w}+\frac{t}{w(w-t)}
$$

we transform (C.1) into

$$
f(t)=\int_{0}^{T} \frac{g(w)}{w} \mathrm{~d} w+t \mathrm{PV} \int_{0}^{T} \frac{w^{y-1} h(w)}{w-t} \mathrm{~d} w
$$

In this equation, the first term of the right hand side is a convergent integral. In order to compute the principal value integral of the second term, we write 


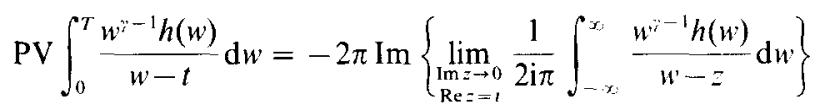

and remark that function $w^{\gamma-1} h(w)$ fulfills the hypotheses of paragraph 29 , Chapter 4 of Mushkelishvili (1953). This reference gives, when $-1<\gamma-1<0$ :

$$
\frac{1}{2 \mathrm{i} \pi} \int_{-,}^{x} \frac{w^{i-1} h(w)}{w-z} \mathrm{~d} w=\frac{\cos (-\gamma \pi)+\mathrm{i} \sin (\gamma \pi)}{2 \mathrm{i} \sin (-\gamma \pi)} h(t)(z-t)+O\left((z-t)^{\prime \prime}\right) \text { with } \gamma_{0}>i,
$$

so that expression (C.4) amounts to

$$
\pi \tan (\pi(\gamma-1 / 2)) h(0) t^{\prime}
$$

For $\gamma-1=0$, Mushkelishvili (1953) provides

$$
\frac{1}{2 \mathrm{i} \pi} \int_{-\infty}^{\infty} \frac{h(w)}{w-z} \mathrm{~d} w=\frac{1}{2 \mathrm{i} \pi} h(t) \ln \frac{1}{z-t},
$$

which leads to express (C.4) as

$$
h(0)|\ln t| .
$$

Establishing the result in case (iii) easily follows from noticing that the polynomial division lcading to equation (C.3) can be iterated to get

$$
f(t)=\sum_{n \rightarrow 0}^{E(\gamma)} t^{n} \int_{0}^{T} w^{\gamma-n-1} h(w) \mathrm{d} w+t^{E(\gamma)+1} \mathrm{PV} \int_{0}^{T} w^{\gamma-E(\eta)-1} \frac{h(w)}{w-t} \mathrm{~d} w,
$$

where $E(\gamma)$ denotes the largest integer strictly smaller than $\gamma$. The last integral can be estimated thanks either to case (ii) or (i) depending upon $\gamma$ being integer or not, which ends the proof for case (iii). To adapt these results around the right bound $t=T$, one just has to use the identity:

$$
\frac{1}{u-t}=\sum_{n=0}^{N} \frac{(t-T)^{n}}{(u-T)^{n+1}}+\frac{(t-T)^{N+1}}{(u-t)(u-T)^{N+1}}
$$

which holds for any non-negative integer $N$. 